TRANSACTIONS OF THE

AMERICAN MATHEMATICAL SOCIETY

Volume 363, Number 2, February 2011, Pages 763-800

S 0002-9947(2010)05286-9

Article electronically published on September 20, 2010

\title{
ENDPOINT ESTIMATES \\ FOR BILINEAR OSCILLATORY INTEGRAL OPERATORS RELATED TO RESTRICTION TO THE CONE
}

\author{
JUNGJIN LEE
}

\begin{abstract}
We prove an endpoint estimate for oscillatory integral operators whose phase function satisfies the cinematic curvature condition. It is a generalization of a result due to Tao (2001).
\end{abstract}

\section{INTRODUCTION}

Fix a dimension $n \geq 2$. Let an oscillatory integral operator $\mathcal{W}^{\lambda}$, depending on a positive parameter $\lambda$, be defined by

$$
\mathcal{W}^{\lambda} f(z):=\int_{\mathbb{R}^{n}} e^{i \lambda \Psi(z, \xi)} a(z, \xi) f(\xi) d \xi, \quad z=(x, t) \in \mathbb{R}^{n} \times \mathbb{R} .
$$

Here, $a \in C_{0}^{\infty}\left(\mathbb{R}^{n+1} \times \mathbb{R}^{n}\right)$ is a fixed smooth cutoff function of compact support in $z$ and $\xi$. The operator $\mathcal{W}^{\lambda}$ can be thought of as a generalization of restriction operators (see [12, 15]), and it appears naturally in the regularity problem of Fourier integral operators (see [11, 14]).

We will consider the $\left(L^{2} \times L^{2} \rightarrow L^{p}\right)$-bilinear estimate

$$
\left\|\mathcal{W}_{1}^{\lambda} f \mathcal{W}_{2}^{\lambda} g\right\|_{L^{p}\left(\mathbb{R}^{n+1}\right)} \leq A \lambda^{-\frac{n+1}{p}}\|f\|_{2}\|g\|_{2}
$$

under certain conditions for the phase functions $\Psi_{1}$ and $\Psi_{2}$, where $\mathcal{W}_{j}^{\lambda}$ is the oscillatory integral operator defined with $a_{j}, \Psi_{j}$ in place of $a, \Psi$, respectively, and $A$ is a constant independent of $\lambda, f$ and $g$.

Following [11, 13, 14] we say that the phase $\Psi$ satisfies the cinematic curvature condition if the following three conditions hold:

- The phase $\Psi(x, t, \xi)$ is real-valued, homogeneous of degree one in $\xi$ and smooth when $\xi \neq 0$.

$$
\left\{\begin{array}{l}
\operatorname{rank} \nabla_{x, \xi}^{2} \Psi=n \\
\partial_{t} \Psi \neq 0 \text { if } \xi \neq 0
\end{array}\right.
$$

Received by the editors March 22, 2009.

2010 Mathematics Subject Classification. Primary 42B20, 35S30.

Key words and phrases. Fourier restriction theorem, oscillatory integral operator.

(C)2010 American Mathematical Society Reverts to public domain 28 years from publication 
- It follows from the above conditions and the implicit function theorem that there exists a function $q(z, \xi)$, homogeneous of degree 1 in $\xi$, such that

$$
\partial_{t} \Psi(z, \xi)=q\left(z, \nabla_{x} \Psi(z, \xi)\right) .
$$

The last condition is that

$$
\operatorname{rank} \nabla_{\xi}^{2} q=n-1 .
$$

A representative model of $\Psi$ satisfying the cinematic curvature condition is

$$
\Psi_{\text {cone }}(x, t, \xi)=x \cdot \xi+t|\xi| .
$$

The corresponding operator $\mathcal{W}_{c}^{\lambda}$ is essentially the Fourier extension operator for the cone, i.e. the adjoint of the Fourier restriction operator related to the cone. Under the assumption that the normal vectors to the surface $\left\{(\xi,|\xi|): \xi \in \operatorname{supp}_{\xi} a_{1}\right\}$ are transverse to the normal vectors to $\left\{(\xi,|\xi|): \xi \in \operatorname{supp}_{\xi} a_{2}\right\}$, Bourgain [3] first showed that when $n=2$ the operator $\mathcal{W}_{c}^{\lambda}$ satisfies the estimate (1.1) for $p>2-\varepsilon$ with some $\varepsilon>0$, and Tao and Vargas [18] obtained the concrete range $p>2-\frac{8}{121}$. In [23], Wolff proved that $\mathcal{W}_{c}^{\lambda}$ satisfies (1.1) in the nearly sharp range $p>\frac{n+3}{n+1}$ for all dimensions $n$. Later, Tao [20] obtained the endpoint result for $p=\frac{n+3}{n+1}$.

Wolff's $\left(L^{2} \times L^{2} \rightarrow L^{p}\right)$-bilinear estimate for $\mathcal{W}_{c}^{\lambda}$ has been extended by S.H. Lee 7. 8. to cover the oscillatory integral operator $\mathcal{W}^{\lambda}$, whose phase functions $\Psi_{1}$ and $\Psi_{2}$ satisfy the cinematic curvature condition and the following separation condition: For each $j=1,2$, we have

$$
\left|\left\langle\frac{\nabla_{x} \Psi_{j}\left(z, \xi_{j}\right)}{\left|\nabla_{x} \Psi_{j}\left(z, \xi_{j}\right)\right|}, \nabla_{\xi} q_{2}\left(z, \nabla_{x} \Psi_{2}\left(z, \xi_{2}\right)\right)-\nabla_{\xi} q_{1}\left(z, \nabla_{x} \Psi_{1}\left(z, \xi_{1}\right)\right)\right\rangle\right| \sim 1
$$

for all $\left(z, \xi_{1}\right) \in \operatorname{supp} a_{1}$ and $\left(z, \xi_{2}\right) \in \operatorname{supp} a_{2}$. (Here the symbol " 1" means the expression on the left is bounded above and below by two uniform constants; see the notation below in this section.)

This condition guarantees that the sets

$$
\begin{gathered}
\left\{\nabla_{\xi} q_{1}\left(z_{1}, \nabla_{x} \Psi_{1}\left(z_{1}, \xi_{1}\right)\right):\left(z_{1}, \xi_{1}\right) \in \operatorname{supp} a_{1}\right\} \quad \text { and } \\
\left\{\nabla_{\xi} q_{2}\left(z_{2}, \nabla_{x} \Psi_{2}\left(z_{2}, \xi_{2}\right)\right):\left(z_{2}, \xi_{2}\right) \in \operatorname{supp} a_{2}\right\}
\end{gathered}
$$

are separated by a distance comparable to 1 . This is a kind of transversality condition and, roughly speaking, its role is to alleviate a certain critical situation by preventing the generation of some complicated Kakeya-type sets. So the bilinear estimate with this condition is expected to be better than the estimate without it (see [7, 18]). (In the case of $\Psi_{\text {cone, }}$, the condition (1.5) implies that the normal vectors to the surface $\left\{(\xi,|\xi|): \xi \in \operatorname{supp}_{\xi} a_{1}\right\}$ are transverse to the normal vectors to $\left\{(\xi,|\xi|): \xi \in \operatorname{supp}_{\xi} a_{2}\right\}$.)

The purpose of this paper is to prove the following theorem.

Theorem 1.1. Let $S_{j}$ be a neighborhood of the support of $a_{j}$ for $j=1,2$. Suppose that $\Psi_{1}$ and $\Psi_{2}$ satisfy a cinematic curvature condition on $S_{1}$ and $S_{2}$, respectively. If $\Psi_{1}, \Psi_{2}$ have (1.5) for all $\left(z, \xi_{1}\right) \in S_{1}$ and $\left(z, \xi_{2}\right) \in S_{2}$, then for $p \geq \frac{n+3}{n+1}$, we have

$$
\left\|\mathcal{W}_{1}^{\lambda} f \mathcal{W}_{2}^{\lambda} g\right\|_{p} \leq A \lambda^{-\frac{n+1}{p}}\|f\|_{2}\|g\|_{2},
$$

where $A$ is a constant which is independent of $\lambda, f$ and $g$. 
This is the $\lambda^{\varepsilon}$-removal version of Theorem 1.2 in [8]. It is an endpoint estimate in the sense that the estimate (1.6) is false if $p<\frac{n+3}{n+1}$. This range of exponent $p$ is found by means of the squashed caps and the one-sheeted hyperboloid examples (see 18 ).

In [20], the endpoint estimate (1.6) for $\mathcal{W}_{c}^{\lambda}$ is used to develop the sharp null form estimates for the solutions to the wave equation, after some generalizations. So, it is expected that Theorem 1.1 also has some applications to the null form estimates for some related equations (see [9, 19, 20, ).

Let us now discuss the oscillatory integral satisfying the Carleson-Sjölin condition. (For a statement of the Carleson-Sjölin condition, see [14, 15.) One of the representative model phases is

$$
\Psi_{\text {parab }}(x, t, \xi)=x \cdot \xi+t|\xi|^{2},
$$

which is related to a paraboloid. The corresponding operator $\mathcal{W}_{p}^{\lambda}$ is essentially the adjoint Fourier restriction operator for the paraboloid which has been studied along with $\mathcal{W}_{c}^{\lambda}$.

When $n=1$, the estimate (1.1) for the oscillatory integral operator $\mathcal{W}^{\lambda}$ satisfying the Carleson-Sjölin condition is well understood due to the works of L. Carleson and P. Sjölin [5] and Hörmander [6].

For $n \geq 2$, the nearly sharp $\left(L^{2} \times L^{2} \rightarrow L^{p}\right)$-bilinear estimate for $\mathcal{W}_{p}^{\lambda}$ was obtained by Tao [21] under a similar separation condition, namely that the normal vectors to the surface $\left\{\left(\xi,|\xi|^{2}\right): \xi \in \operatorname{supp}_{\xi} a_{1}\right\}$ are transverse to the normal vectors to $\left\{\left(\xi,|\xi|^{2}\right): \xi \in \operatorname{supp}_{\xi} a_{2}\right\}$. This has been generalized for the surfaces with principal curvatures of different signs by S.H. Lee [7, 8] and independently by A. Vargas [22]. S.H. Lee [8] showed that the oscillatory integral operators satisfying the CarlesonSjölin condition satisfy (1.6) with a $\lambda^{\varepsilon}$-loss under the following separation condition: For any $\left(z, \xi_{1}\right) \in \operatorname{supp} a_{1}$ and $\left(z, \xi_{2}\right) \in \operatorname{supp} a_{2}$,

$$
\left|\left\langle H_{j}\left(z, \xi_{j}\right) \delta\left(z, \xi_{1}, \xi_{2}\right), H_{j}^{-1}\left(z, \xi_{j}\right)\left[\nabla_{\xi, \xi}^{2} q_{j}\left(z, \nabla_{x} \Psi_{j}\left(z, \xi_{j}\right)\right)\right]^{-1} \delta\left(z, \xi_{1}, \xi_{2}\right)\right\rangle\right| \sim 1,
$$

where $H_{j}(z, \xi)=\nabla_{x, \xi}^{2} \Psi_{j}(z, \xi)$ and

$$
\delta\left(z, \xi_{1}, \xi_{2}\right)=\nabla_{\xi} q_{1}\left(z, \nabla_{x} \Psi_{1}\left(z, \xi_{1}\right)\right)-\nabla_{\xi} q_{2}\left(z, \nabla_{x} \Psi_{2}\left(z, \xi_{2}\right)\right) .
$$

But, the $\lambda^{\varepsilon}$-removed version (or the endpoint estimate) remains open even for the model operator $\mathcal{W}_{p}^{\lambda}$. Although $\mathcal{W}_{c}^{\lambda}$ and $\mathcal{W}_{p}^{\lambda}$ share many properties, the method of proof in [20] breaks down, because the strong Huygens' principle fails for $\mathcal{W}_{p}^{\lambda}$. An endpoint estimate for $\mathcal{W}_{p}^{\lambda}$ may play an important role for improving the null form estimates for the wave equations (see [9, 20]).

Notation. Let $C_{0}$ be an initial constant picked at the beginning of our arguments, which does not depend on anything. Let $N$ be a large number depending only on $n$, and let $\varepsilon$ be an arbitrary small positive number. But, both $N$ and $1 / \varepsilon$ are much smaller than $C_{0}$. We will use $N$ and $\varepsilon$ as exponents such as $\lambda^{-N}$ and $\lambda^{\varepsilon}$. Let $C$ denote various large numbers that vary from line to line, which possibly depend on $N$ and $\varepsilon$ but do not depend on $C_{0}$. Let $A \lesssim B$ or $A=\mathcal{O}(B)$ denote the estimate $A \leq C B$ and let $A \ll B$ denote $A<C^{-1} \widetilde{B}$. The $\lesssim$ notation will be used to deal with error terms. Let $A \sim B$ mean that $A \lesssim B$ and $B \lesssim A$. To make a factor of the main term precise, we use a small parameter $0<c \ll 1$ which may be optimized later. We will get many estimates of the form $A \leq(1+C N c) \mathcal{M}+C c^{-C} \mathcal{E}$, where 
$\mathcal{M}$ is the main term and $\mathcal{E}$ is the error term. In our arguments, it is important that the factor of the main term is sufficiently close to 1 .

We define some geometric objects. We use $D_{r}=D_{r}^{z_{0}}$ to denote the disc of radius $r$ and centered at $z_{0}=\left(x_{0}, t_{0}\right)$, i.e.

$$
D_{r}^{z_{0}}=\left\{\left(x, t_{0}\right):\left|x-x_{0}\right| \leq r\right\} .
$$

Let $\gamma_{j}^{v}(t)$ be a curve satisfying the differential equation

$$
\frac{d}{d t} \gamma_{j}^{v}(t)=-\nabla_{\xi} q_{j}\left(\gamma_{j}^{v}(t), t, \nabla_{x} \Psi_{j}\left(\gamma_{j}^{v}(t), t, v\right)\right) .
$$

Note that this differential equation is induced from the fact that

$$
\nabla_{\xi} \Psi_{j}\left(\gamma^{v}\left(t_{1}\right), t_{1}, v\right)=\nabla_{\xi} \Psi_{j}\left(\gamma^{v}\left(t_{2}\right), t_{2}, v\right)
$$

for any $t_{1}, t_{2} \in \mathbb{R}$. We define the conic surface $\Lambda_{j}^{\lambda}=\Lambda_{j}^{\lambda, z_{0}}$ with vertex $z_{0}=\left(x_{0}, t_{0}\right)$, generated by the phase $\Psi_{j}$, as

$$
\Lambda_{j}^{\lambda, z_{0}}=\bigcup_{v \in \operatorname{supp}_{\xi} a_{j}}\left\{\left(\lambda \gamma_{j}^{v}(t), \lambda t\right): t \in \mathbb{R}\right\}, \quad j=1,2,
$$

where $\gamma_{j}^{v}(t)$ satisfies that $\gamma_{j}^{v}\left(t_{0} / \lambda\right)=x_{0} / \lambda$.

We use $\Lambda_{j}^{\lambda}(r)=\Lambda_{j}^{\lambda, z_{0}}(r)$ to denote the $r$-neighborhood of $\Lambda_{j}^{\lambda, z_{0}}$ for any $r>0$ and let

$$
\Lambda^{\lambda, z}:=\Lambda_{1}^{\lambda, z} \cup \Lambda_{2}^{\lambda, z}
$$

Sometimes we will not indicate $\lambda$ when it is clear from the context.

\section{Outline of the proof of Theorem 1.1. Let}

$$
\mathcal{G}_{j}^{\lambda} f(z):=\int e^{i \Psi_{j}^{\lambda}(z, \xi)} a_{j}^{\lambda}(z, \xi) \widehat{f}(\xi) d \xi
$$

and

$$
\Psi_{j}^{\lambda}(z, \xi)=\lambda \Psi_{j}(z / \lambda, \xi), \quad a_{j}^{\lambda}(z, \xi)=a_{j}(z / \lambda, \xi) .
$$

By scaling, the estimate (1.6) is equivalent to

$$
\left\|\mathcal{G}_{1}^{\lambda} f \mathcal{G}_{2}^{\lambda} g\right\|_{p} \leq A\|f\|_{2}\|g\|_{2} .
$$

We will deal with (1.10) instead of (1.6) throughout the argument. For the sake of convenience, let us write $U_{j}^{\lambda}$ for $\mathcal{G}_{j}^{\lambda} f$, and we may assume that

$$
\|f\|_{2}=\|g\|_{2}=1 \text {. }
$$

By interpolation it suffices to show (1.10) for $p=\frac{n+3}{n+1}$, so we will take $p=\frac{n+3}{n+1}$ throughout the proof.

We will basically follow Tao's arguments in 20. Let us now explain the strategy of the proof heuristically. (So, the following formulas may not be exactly right.) We may assume that $\lambda$ is larger than some big constant, because a trivial estimation gives $\left\|U_{1}^{\lambda} U_{2}^{\lambda}\right\|_{p} \lesssim \lambda^{C}$. So, we assume that $\lambda \geq 2^{C_{0}}$.

The non-endpoint estimate is obtained by an induction-on-scale argument. If $A(\lambda)$ is the best constant such that the estimate

$$
\left\|U_{1}^{\lambda} U_{2}^{\lambda}\right\|_{p} \leq A(\lambda)
$$

holds for $\mathcal{O}(1)$ perturbations of $\Psi_{1}$ and $\Psi_{2}$, the induction-on-scale argument gives the following recursive inequality:

$$
A(\lambda) \lesssim A(\lambda / 2)+\lambda^{C \varepsilon}, \quad \forall \varepsilon>0,
$$


from which we can obtain $A(\lambda) \lesssim \lambda^{C \varepsilon}$. We point out that since (1.12) is not translation-invariant, it is difficult to use (1.12) as in [20, 23.

To remove the $\lambda^{\varepsilon}$-loss, we will improve this recursive inequality by combining a localization property of $U_{j}^{\lambda}$ called Huygens' principle and the energy concentration of $U_{1}^{\lambda}$ and $U_{2}^{\lambda}$. We first find a disc $D$ of radius $r_{e}$ on which the energies of $U_{1}^{\lambda}$ and $U_{2}^{\lambda}$ concentrate at the same time. Indeed, we take a largest disc $D=D_{r_{e}}^{z_{e}}$, $z_{e}=\left(x_{e}, t_{e}\right)$, satisfying

$$
\left\|U_{1}^{\lambda}\right\|_{L^{2}(D)}\left\|U_{2}^{\lambda}\right\|_{L^{2}(D)} \leq(1-\delta)\left\|U_{1}^{\lambda}\left(t_{e}\right)\right\|_{2}\left\|U_{2}^{\lambda}\left(t_{e}\right)\right\|_{2},
$$

where $0<\delta \ll 1$ is properly chosen. Then we can show that

$$
\left\|U_{1}^{\lambda} U_{2}^{\lambda}\right\|_{p} \leq(1+C N c)\left\|U_{1}^{\lambda} U_{2}^{\lambda}\right\|_{L^{p}\left(Q^{e} \cap \Lambda^{\lambda, z_{e}}\left(r_{e}\right)\right)}+2^{C C_{0}},
$$

where $Q^{e}$ is the cube of side-length $r_{e}^{2}$ with center $z_{e}$. So, we apply the inductionon-scale argument to $\left\|U_{1}^{\lambda} U_{2}^{\lambda}\right\|_{L^{p}\left(Q^{e} \cap \Lambda^{\lambda, z_{e}}\left(r_{e}\right)\right)}$ instead of $\left\|U_{1}^{\lambda} U_{2}^{\lambda}\right\|_{p}$. For this, we introduce a new induction hypothesis. Let $A^{\lambda}(R, r)$ be the best constant such that the inequality

$$
\left\|U_{1}^{\lambda} U_{2}^{\lambda}\right\|_{L^{p}\left(Q_{R} \cap \Lambda^{\lambda, z_{e}}(r)\right)} \leq A^{\lambda}(R, r)\left(\left\|U_{1}^{\lambda}\left(t_{e}\right)\right\|_{2}\left\|U_{2}^{\lambda}\left(t_{e}\right)\right\|_{2}\right)^{1 / p} E_{r}\left(U_{1}^{\lambda}, U_{2}^{\lambda}\right)^{1 / p^{\prime}}
$$

holds for any cube $Q_{R}$ in $\mathbb{R}^{n+1}$, where

$$
E_{r}\left(U_{1}^{\lambda}, U_{2}^{\lambda}\right)=\max \left\{\frac{1}{2}\left\|U_{1}^{\lambda}\left(t_{e}\right)\right\|_{2}\left\|U_{2}^{\lambda}\left(t_{e}\right)\right\|_{2},\left\|U_{1}^{\lambda}\right\|_{L^{2}\left(D_{r}^{z e}\right)}\left\|U_{2}^{\lambda}\right\|_{L^{2}\left(D_{r}^{z e}\right)}\right\} .
$$

Through the induction-on-scale argument, we can obtain the recursive inequality

$$
A^{\lambda}(R, r) \leq\left(1+C C_{0}^{-C}\right) A^{\lambda}(R / 2, r)+(R / r)^{-C} 2^{C C_{0}} .
$$

Note that here we need a more delicate analysis to obtain the factor $\left(1+C C_{0}^{-C}\right)$ than in 8 . In particular, we cannot use the pigeonhole principle as in 8 . The factor $(R / r)^{-C}$ will be obtained from exploiting the fact that $\Lambda^{\lambda}$ is a conic hypersurface, and it compensates for the $R^{\varepsilon}$-loss caused by an iteration of the above inequality. After iterating to the scale $r$, we have

$$
A^{\lambda}(R, r) \leq\left(1+C C_{0}^{-C}\right) A^{\lambda}(r, r)+C 2^{C C_{0}} .
$$

Combining this with (1.14) and (1.15), we thus have

$$
\left\|U_{1}^{\lambda} U_{2}^{\lambda}\right\|_{p} \leq(1+C N c)\left(1+C C_{0}^{-C}\right)(1-\delta) A^{\lambda}\left(r_{e}, r_{e}\right)\left\|U_{1}^{\lambda}\left(t_{e}\right)\right\|_{2}\left\|U_{2}^{\lambda}\left(t_{e}\right)\right\|_{2}+2^{C C_{0}} .
$$

Abusing the notation, we set $A(\lambda)$ to be the best constant such that

$$
\left\|U_{1}^{\lambda} U_{2}^{\lambda}\right\|_{p} \leq A(\lambda)\left\|U_{1}^{\lambda}\left(t_{e}\right)\right\|_{2}\left\|U_{2}^{\lambda}\left(t_{e}\right)\right\|_{2} .
$$

Then we have

$$
A(\lambda) \leq(1+C N c)\left(1+C C_{0}^{-C}\right)(1-\delta) A^{\lambda}\left(r_{e}, r_{e}\right)+2^{C C_{0}} .
$$

By the concentration property (1.13), we can get

$$
A^{\lambda}\left(r_{e}, r_{e}\right) \leq\left(1+C C_{0}^{-C}\right) A(\lambda)+2^{C C_{0}} .
$$

So, we finally obtain

$$
A(\lambda) \leq(1+C N c)\left(1+C C_{0}^{-C}\right)(1-\delta) A(\lambda)+2^{C C_{0}} .
$$


By taking suitably small $\delta$ and $c$, we can get the estimate

$$
A(\lambda) \leq\left(1-C C_{0}^{-C}\right) A(\lambda)+2^{C C_{0}} .
$$

This would finally yield $A(\lambda) \leq 2^{C C_{0}}$.

Organization of the paper. The paper is organized as follows. In the next section, we derive an $L^{2}$-estimate of $U_{j}^{\lambda}$ on a neighborhood of the conic surface $\Lambda_{k}^{\lambda}$ that is generated by $\Psi_{k}$ for $j, k=1,2$ and $j \neq k$. This is a basic estimate in our argument, which is used in several places, in particular, in section 4 to prove the main proposition. Here, the separation condition is a key condition. In section 3, we recall the wave packet decomposition and state the main proposition. The wave packet decomposition is a fundamental tool which has been used to get improvements in the Fourier restriction problems and related problems. In section 4 , we give the proof of the main proposition by interpolating $L^{2} \times L^{2} \rightarrow L^{1}$ and $L^{2} \times L^{2} \rightarrow L^{2}$ estimates. In section 5, using the main proposition and the inductionon-scale argument we prove the $\lambda^{\varepsilon}$-loss version of Theorem 1.1, which is the same as Theorem $1.2 \mathrm{in} 8$. The result in this section is not used later. In section 6 , we develop some machinery for the localization of $U_{j}^{\lambda}$. We define an approximation $\mathcal{U}_{j}^{\lambda}$ of $U_{j}^{\lambda}$ and define a localization operator $L_{D} \mathcal{U}_{j}^{\lambda}$ of $\mathcal{U}_{j}^{\lambda}$. After that, we give several estimates for $L_{D} \mathcal{U}_{j}^{\lambda}$. In section 7 , we localize $\mathcal{U}_{1}^{\lambda} \mathcal{U}_{2}^{\lambda}$ to the energy concentrated cube $Q^{e}$ and also to the neighborhood of the conic surface $\Lambda^{\lambda, z_{e}}$ whose vertex $z_{e}$ is in the energy concentrated portion. In section 8 , we define a new wave packet, which is essentially the same as the previous one in section 3 . Here, we will show that the new one also satisfies the properties in section 3 . The reason why we work with new wave packets is to obtain more accurate constants in the next section. In section 9 , we apply the induction-on-scale argument to $\left\|\mathcal{U}_{1}^{\lambda} \mathcal{U}_{2}^{\lambda}\right\|_{L^{p}\left(Q^{e} \cap \Lambda^{\lambda, z_{e}}\left(r_{e}\right)\right)}$. In section 10, we finish the proof of Theorem 1.1 by combining the propositions obtained in sections 7 and 9. Finally, in an Appendix, we explain a stationary phase method which is used repeatedly in our paper.

\section{2. $L^{2}$-ESTIMATE ON A CONIC SURFACE}

In the following proposition, we will use the geometric property that any curve $\left(\lambda \gamma_{j}^{v}(t), \lambda t\right)$ for $v \in \operatorname{supp}_{\xi} a_{j}$ intersects $\Lambda_{k}^{\lambda}$ in at most one point for $j \neq k$ and $\lambda \geq 0$. This property is due to the separation condition (1.5) and the curvature condition (1.4). (For the necessity of the separation condition, see [7, 22.) We begin with the following lemma, which is needed to utilize the curvature condition.

Lemma 2.1. Let $h(x)$ be a smooth function on a domain $K \subset \mathbb{R}^{n}$. Suppose that $h(x)$ is homogeneous of degree one. Then for any $x, x^{\prime} \in K$, we have

$$
\left\langle\frac{x}{|x|}, \nabla h\left(x^{\prime}\right)-\nabla h(x)\right\rangle=\mathcal{O}\left(\left|\frac{x^{\prime}}{\left|x^{\prime}\right|}-\frac{x}{|x|}\right|^{2}\right) .
$$

Proof. Since $h(x)$ is homogeneous of degree one, $\nabla h$ is homogeneous of degree zero, i.e. $\nabla h(r x)=\nabla h(x)$ for all $r \in \mathbb{R}$, which means that the radial directional derivative of $\nabla h$ always vanishes:

$$
(x /|x|)^{T} \nabla^{2} h(x)=\mathbf{0} .
$$


We have

$$
\begin{aligned}
\nabla h\left(x^{\prime}\right)-\nabla h(x) & =\nabla h\left(\frac{x^{\prime}}{\left|x^{\prime}\right|}\right)-\nabla h\left(\frac{x}{|x|}\right) \\
& =\nabla^{2} h\left(\frac{x}{|x|}\right)\left(\frac{x^{\prime}}{\left|x^{\prime}\right|}-\frac{x}{|x|}\right)+\mathcal{O}\left(\left|\frac{x^{\prime}}{\left|x^{\prime}\right|}-\frac{x}{|x|}\right|^{2}\right) .
\end{aligned}
$$

Taking the inner product with $\frac{x}{|x|}$, we obtain (2.1).

Proposition 2.2. Let $\Psi_{1}$ and $\Psi_{2}$ satisfy a cinematic curvature condition on supp $a_{1}$ and supp $a_{2}$, respectively. Suppose that $\Psi_{1}$ and $\Psi_{2}$ satisfy (1.5) on supp $a_{1}$ and supp $a_{2}$. Then we have

$$
\left\|\mathcal{G}_{j}^{\lambda} f\right\|_{L^{2}\left(\Lambda_{k}^{\lambda}(r)\right)} \lesssim r^{1 / 2}\|f\|_{2}
$$

for $j, k=1,2$ and $j \neq k$.

Proof. We rewrite the estimate as

$$
\int\left\|\chi_{\Lambda_{k}^{\lambda}(r)}(t)\left[\mathcal{G}_{j}^{\lambda}(t)\right] f\right\|_{L_{x}^{2}\left(\mathbb{R}^{n}\right)}^{2} d t \lesssim r\|f\|_{L^{2}\left(\mathbb{R}^{n}\right)}^{2} .
$$

By the Riesz representation theorem, we have

$$
\iint \chi_{\Lambda_{k}^{\lambda}(r)}(x, t)\left[\mathcal{G}_{j}^{\lambda}(t)\right] f(x) \overline{g(x, t)} d x d t \lesssim r^{1 / 2}\|f\|_{L^{2}\left(\mathbb{R}^{n}\right)}\|g\|_{L^{2}\left(\mathbb{R}^{n+1}\right)} .
$$

Let us rewrite the left side of the above inequality as

$$
\int\left\langle\left[\mathcal{G}_{j}^{\lambda}(t)\right] f, \chi_{\Lambda_{k}^{\lambda}(r)}(t) g(t)\right\rangle d t
$$

By duality, we have

$$
\int\left\langle f,\left[\mathcal{G}_{j}^{\lambda}(t)\right]^{*}\left(\chi_{\Lambda_{k}^{\lambda}(r)}(t) g(t)\right)\right\rangle d t=\left\langle f, \int\left[\mathcal{G}_{j}^{\lambda}(t)\right]^{*}\left(\chi_{\Lambda_{k}^{\lambda}(r)}(t) g(t)\right) d t\right\rangle .
$$

So we have

$$
\left\|\int\left[\mathcal{G}_{j}^{\lambda}(t)\right]^{*}\left(\chi_{\Lambda_{k}^{\lambda}(r)}(t) g(t)\right) d t\right\|_{L^{2}\left(\mathbb{R}^{n}\right)}^{2} \lesssim r\|g\|_{L^{2}\left(\mathbb{R}^{n+1}\right)}^{2} .
$$

By rescaling it suffices to show that

$$
\iiint \int K(x, t, y, s) g(x, t) \overline{g(y, s)} d t d s d x d y \lesssim \lambda^{-n-1} r\|g\|_{L^{2}\left(\mathbb{R}^{n+1}\right)}^{2},
$$

where

$$
\begin{aligned}
K(x, t, y, s)=\chi_{\Lambda_{k}^{1}\left(\lambda^{-1} r\right)}(x, t) \chi_{\Lambda_{k}^{1}\left(\lambda^{-1} r\right)}(y, s) & \\
& \times \int e^{i \lambda\left(\Psi_{j}(y, s, \xi)-\Psi_{j}(x, t, \xi)\right)} a_{j}(x, t, \xi) a_{j}(y, s, \xi) d \xi .
\end{aligned}
$$

We claim that

$$
|K(x, t, y, s)| \lesssim(1+\lambda|x-y|)^{-N_{1}}\left(1+C \lambda r^{-1}|t-s|\right)^{-N_{2}},
$$

for any $N_{1}, N_{2}>0$.

Assuming this claim, the left side of (2.2) is

$$
\lesssim \iiint \int \frac{g(x, t) \overline{g(y, s)}}{(1+\lambda|x-y|)^{N_{1}}\left(1+C \lambda r^{-1}|t-s|\right)^{N_{2}}} d x d y d t d s .
$$


Applying Young's inequality twice, we obtain that the left side of (2.2) is

$$
\begin{aligned}
& \lesssim \lambda^{-n} \iint \frac{1}{\left(1+C \lambda r^{-1}|t-s|\right)^{N_{2}}}\|g(t)\|_{2}^{2}\|g(s)\|_{2}^{2} d t d s \\
& \lesssim \lambda^{-n-1} r\|g\|_{2}^{2} .
\end{aligned}
$$

Thus, it suffices to show the claim (2.3). Consider the phase of the kernel $K$. Expand the phase

$$
\begin{aligned}
& \Psi_{j}(x, t, \xi)-\Psi_{j}(y, s, \xi) \\
& =\left(\Psi_{j}(x, t, \xi)-\Psi_{j}(x, s, \xi)\right)+\left(\Psi_{j}(x, s, \xi)-\Psi_{j}(y, s, \xi)\right) \\
& =(t-s) \partial_{t} \Psi_{j}(x, s, \xi)+(x-y) \nabla_{x} \Psi_{j}(y, s, \xi)+\mathcal{O}\left(|t-s|^{2}\right)+\mathcal{O}\left(|x-y|^{2}\right) .
\end{aligned}
$$

Differentiating in the $\xi$-variables, we have

$$
\begin{aligned}
\nabla_{\xi}\left[\Psi_{j}(x, t, \xi)-\Psi_{j}(y, s, \xi)\right] & \\
=(t-s) \nabla_{\xi} \partial_{t} \Psi_{j}(x, s, \xi)+(x-y) & \nabla_{\xi, x}^{2} \Psi_{j}(y, s, \xi) \\
& +\mathcal{O}\left(|t-s|^{2}\right)+\mathcal{O}\left(|x-y|^{2}\right)
\end{aligned}
$$

Since both $(x, t)$ and $(y, s)$ are in $\Lambda_{k}^{1}\left(\lambda^{-1} r\right)$, we have

$$
\begin{aligned}
& x=\gamma_{k}^{v}(t)+\mathcal{O}\left(\lambda^{-1} r\right), \\
& y=\gamma_{k}^{v^{\prime}}(s)+\mathcal{O}\left(\lambda^{-1} r\right),
\end{aligned}
$$

for some $v, v^{\prime} \in \operatorname{supp}_{\xi} a_{k}$. Let $z_{0}=\left(x_{0}, t_{0}\right)$ be the center of $\Lambda_{k}^{1}$. Expanding $\gamma_{j}^{v}$, we have

We thus have

$$
\gamma_{j}^{v}(t)=x_{0}-\left(t-t_{0}\right) \nabla_{\xi} \partial_{t} \Psi_{j}\left(z_{0}, v\right)+\mathcal{O}\left(\left|t-t_{0}\right|^{2}\right) .
$$

$$
\begin{aligned}
& x-y=-(t-s) \nabla_{\xi} \partial_{t} \Psi_{k}\left(z_{0}, v\right)-(s-\left.t_{0}\right)\left(\nabla_{\xi} \partial_{t} \Psi_{k}\left(z_{0}, v^{\prime}\right)-\nabla_{\xi} \partial_{t} \Psi_{k}\left(z_{0}, v\right)\right) \\
&+\mathcal{O}\left(\left|t-t_{0}\right|^{2}\right)+\mathcal{O}\left(\left|s-t_{0}\right|^{2}\right)+\mathcal{O}\left(\lambda^{-1} r\right) .
\end{aligned}
$$

Inserting this into (2.5), we have

$$
\begin{aligned}
& \nabla_{\xi}\left[\Psi_{j}(x, t, \xi)-\Psi_{j}(y, s, \xi)\right]=(t-s)\left(\nabla_{\xi} \partial_{t} \Psi_{j}(x, s, \xi)-\nabla_{\xi} q_{k}\left(z_{0}, \nabla_{x} \Psi_{k}\left(z_{0}, v\right)\right)\right) \\
& +\left(s-t_{0}\right)\left(\nabla_{\xi} q_{k}\left(z_{0}, \nabla_{x} \Psi_{k}\left(z_{0}, v^{\prime}\right)\right)-\nabla_{\xi} q_{k}\left(z_{0}, \nabla_{x} \Psi_{k}\left(z_{0}, v\right)\right)\right) \\
& \quad+\mathcal{O}\left(\lambda^{-1} r\right)+\mathcal{O}\left(\left|t-t_{0}\right|^{2}\right)+\mathcal{O}\left(\left|s-t_{0}\right|^{2}\right)+\mathcal{O}\left(|t-s|^{2}\right)+\mathcal{O}\left(|x-y|^{2}\right)
\end{aligned}
$$

If the support of $a_{j}$ is sufficiently small, then we have

$$
\begin{aligned}
& \nabla_{\xi}\left[\Psi_{j}(x, t, \xi)-\Psi_{j}(y, s, \xi)\right] \\
& \quad=(t-s)\left(\nabla_{\xi} q_{j}\left(x, s, \nabla_{x} \Psi_{j}(x, s, \xi)\right)-\nabla_{\xi} q_{k}\left(z_{0}, \nabla_{x} \Psi_{k}\left(z_{0}, v\right)\right)\right) \\
& \quad+\left(s-t_{0}\right)\left(\nabla_{\xi} q_{k}\left(z_{0}, \nabla_{x} \Psi_{k}\left(z_{0}, v^{\prime}\right)\right)-\nabla_{\xi} q_{k}\left(z_{0}, \nabla_{x} \Psi_{k}\left(z_{0}, v\right)\right)\right) \\
& +\mathcal{O}\left(\lambda^{-1} r\right) .
\end{aligned}
$$

Take the inner product with $u:=\frac{\nabla_{x} \Psi_{k}\left(x_{0}, t_{0}, v\right)}{\left|\nabla_{x} \Psi_{k}\left(x_{0}, t_{0}, v\right)\right|}$ in (2.6). Then, by (1.3), (1.5) and Lemma 2.1, we obtain

$$
\left|\left\langle u, \nabla_{\xi}\left[\Psi_{j}(x, t, \xi)-\Psi_{j}(y, s, \xi)\right]\right\rangle\right| \gtrsim|t-s|-C \lambda^{-1} r .
$$


Thus by the stationary phase method (see Lemma 11.1) and

$$
|t-s|-C \lambda^{-1} r \gtrsim 1 \Longrightarrow \lambda r^{-1}|t-s| \gtrsim 1,
$$

we see that the kernel $K$ is very small when $|t-s| \gtrsim \lambda^{-1} r$, that is,

$$
|K(x, t, y, s)| \lesssim\left(1+C \lambda r^{-1}|t-s|\right)^{-N_{2}}, \quad \forall N_{2}>0 .
$$

For fixed $s$ and $t$, consider the integral of kernel $K$. By (2.4), we can write

$$
|K(x, t, y, s)|=\left|\int e^{i \lambda(x-y) \nabla_{x} \Psi_{j}(y, s, \xi)} a(x, t, y, s, \xi) d \xi\right|,
$$

for some smooth function $a(x, t, y, s, \xi)$ with compact support. By the first condition in (1.2), the stationary phase method (see Lemma 11.1) gives

$$
|K(x, t, y, s)| \lesssim(1+\lambda|x-y|)^{-N_{1}}, \quad \forall N_{1}>0 .
$$

From (2.7) and (2.8), we obtain the claim (2.3).

\section{MAIN PROPOSITION}

In this section we state the main proposition. (The proof of the proposition will be given in the next section.) Before that, we need to introduce the wave packet decomposition. It is a little different from that in 8 since we need to handle the overlap of Schwartz tails in detail.

Let $C_{0}$ be a very large integer. Fix $R$ with $C_{0}<R \leq \lambda$. Let $0<c \leq 2^{-C_{0}}$ be a constant to be chosen later. Now let $G^{R}=c^{-2} R^{1 / 2} \mathbb{Z}^{n}$ be a spatial lattice and let $V_{j}^{R}=\Xi_{j} \cap R^{-1 / 2} \mathbb{Z}^{n}$ be a frequency lattice for $j=1,2$, where $\Xi_{j}$ is the $\xi$-projection of $S_{j}$. (Here, what we want is to take the separated points in a set. So, we may take $G^{R}\left(x_{0}\right)=c^{-2} R^{1 / 2} \mathbb{Z}^{n}+x_{0}$ and $V_{j}^{R}\left(\xi_{0}\right)=\Xi_{j} \cap\left(R^{-1 / 2} \mathbb{Z}^{n}+\xi_{0}\right)$ for some $x_{0}, \xi_{0} \in \mathbb{R}^{n}$.) Let $\eta$ be a nonnegative Schwartz function whose Fourier transform is supported in a unit disk with $\sum_{k \in \mathbb{Z}^{n}} \eta(\cdot-k)=1$, and let $\rho$ be a nonnegative smooth function which is supported in $B(0,1)$, and satisfies $\sum_{k \in \mathbb{Z}^{n}} \rho(\cdot-k)=1$. Then for each $(y, v) \in G^{R} \times V_{j}^{R}$, we set

$$
\eta_{y}(x)=\eta\left(c^{2} R^{-1 / 2}(x-y)\right) \quad \text { and } \quad \rho_{v}(\xi)=\rho\left(R^{1 / 2}(\xi-v)\right) .
$$

We define a wave packet $u_{(y, v)}$ by

$$
u_{(y, v)}=\mathcal{G}_{j}^{\lambda}\left(f_{(y, v)}\right) .
$$

Here,

$$
f_{(y, v)}=\eta_{y} \mathcal{F}^{-1}\left(\rho_{v} \widehat{f}\right),
$$

where the Fourier support of $f$ is contained in $\Xi_{j}$ and $\mathcal{F}^{-1}$ denotes the inverse Fourier transform. If we set

$$
h_{(y, v)}=R^{n / 4} M\left(\mathcal{F}^{-1}\left(\rho_{v} \widehat{f}\right)\right)(y) \quad \text { and } \quad \phi_{(y, v)}=h_{(y, v)}^{-1} u_{(y, v)},
$$

then we have the decomposition

$$
\mathcal{G}_{j}^{\lambda} f=\sum_{(y, v) \in G^{R} \times V_{j}^{R}} h_{(y, v)} \phi_{(y, v)},
$$

where $M$ denotes the Hardy-Littlewood maximal operator.

The wavepackets have the following properties. 
Lemma 3.1. Suppose that $0<c \leq 2^{-C_{0}}$.

(1) For each $(y, v) \in G^{R} \times V_{j}^{R}$, we have

$$
\left|\phi_{(y, v)}(x, t)\right| \lesssim c^{-C} R^{-n / 4}\left(1+\frac{\left|x-\lambda \gamma_{j}^{v}(t / \lambda)\right|}{R^{1 / 2}}\right)^{-N^{10}},
$$

where $\gamma_{j}^{v}(t)=\gamma_{j}^{(y, v)}(t)$ satisfies $\nabla_{\xi} \Psi_{j}\left(\gamma_{j}^{(y, v)}(0), 0, v\right)=2 \pi y / \lambda$.

(2) If the Fourier support of $f$ is contained in $\operatorname{supp}_{\xi} a_{j}$, then we have

$$
\left(\sum_{(y, v) \in G^{R} \times V_{j}^{R}}\left|h_{(y, v)}\right|^{2}\right)^{1 / 2} \lesssim\|f\|_{2} .
$$

Proof. Let us first show (11). The Fourier support of $f_{(y, v)}$ is contained in the $\left(1+c^{2}\right) R^{-1 / 2}$-neighborhood of $v$. So, we can insert a smooth bump function $\widetilde{\rho}_{v}(\xi)=$ $\rho\left(\left(1+c^{2}\right)^{-1} R^{1 / 2}(\xi-v)\right)$ into $\mathcal{G}_{j}^{\lambda} f_{(y, v)}$. By a rearrangement we may write

$$
u_{(y, v)}(z)=\mathcal{G}_{j}^{\lambda} f_{(y, v)}(z)=\int K_{v}\left(z, x^{\prime}\right) f_{(y, v)}\left(x^{\prime}\right) d x^{\prime},
$$

where

$$
K_{v}\left(z, x^{\prime}\right)=\int e^{i\left(\Psi_{j}^{\lambda}(z, \xi)-2 \pi x^{\prime} \cdot \xi\right)} a_{j}^{\lambda}(z, \xi) \widetilde{\rho}_{v}(\xi) d \xi .
$$

Applying the stationary phase method (see Lemma 11.1), we get

$$
\left|K_{v}\left(z, x^{\prime}\right)\right| \lesssim\left(\left(1+c^{2}\right) R^{-1 / 2}\right)^{n}\left(1+\frac{\left(1+c^{2}\right)\left|\lambda \nabla_{\xi} \Psi_{j}(z / \lambda, v)-2 \pi x^{\prime}\right|}{R^{1 / 2}}\right)^{-N^{10}} .
$$

Thus, we have

$$
\begin{aligned}
& \left|u_{(y, v)}(z)\right| \\
& \lesssim\left(\left(1+c^{2}\right) R^{-1 / 2}\right)^{n} \int\left(1+\frac{\left|\lambda \nabla_{\xi} \Psi_{j}(z / \lambda, v)-2 \pi x^{\prime}\right|}{\left(1+c^{2}\right)^{-1} R^{1 / 2}}\right)^{-N^{10}} \eta_{y}\left(x^{\prime}\right) \mathcal{F}^{-1}\left(\rho_{v} \widehat{f}\right)\left(x^{\prime}\right) d x^{\prime} \\
& \lesssim c^{-C}\left(1+\frac{\left|\lambda \nabla_{\xi} \Psi_{j}(z / \lambda, v)-2 \pi y\right|}{2 c^{-2} R^{1 / 2}}\right)^{-N^{10}} M\left(\mathcal{F}^{-1}\left(\rho_{v} \widehat{f}\right)\right)(y) .
\end{aligned}
$$

Since $0<c \leq 2^{-C_{0}}$ and $N$ is much smaller than $C_{0}$, we have

$$
\left(1+\frac{\left|\lambda \nabla_{\xi} \Psi_{j}(z / \lambda, v)-2 \pi y\right|}{2 c^{-2} R^{1 / 2}}\right)^{-N^{10}} \lesssim c^{-C}\left(1+\frac{\left|\lambda \nabla_{\xi} \Psi_{j}(z / \lambda, v)-2 \pi y\right|}{R^{1 / 2}}\right)^{-N^{10}} .
$$

From the initial condition $\nabla_{\xi} \Psi_{j}\left(\gamma_{j}^{v}(0), 0, v\right)=2 \pi y / \lambda$ and (1.8), we have

$$
\begin{aligned}
\left|\nabla_{\xi} \Psi_{j}(z / \lambda, v)-2 \pi y / \lambda\right| & =\left|\nabla_{\xi} \Psi_{j}(z / \lambda, v)-\nabla_{\xi} \Psi_{j}\left(\gamma_{j}^{v}(t / \lambda), t / \lambda, v\right)\right| \\
& \sim\left|x / \lambda-\gamma_{j}^{v}(t / \lambda)\right| .
\end{aligned}
$$

We thus obtain

$$
\left|u_{(y, v)}(x, t)\right| \lesssim c^{-C} M\left(\mathcal{F}^{-1}\left(\rho_{v} \widehat{f}\right)\right)(y)\left(1+\frac{\left|x-\lambda \gamma_{j}^{v}(t / \lambda)\right|}{R^{1 / 2}}\right)^{-N^{10}},
$$

from which we get (1).

Next we show (2). By the uncertainty principle, if $\left|x-x^{\prime}\right| \lesssim c^{-2} R^{1 / 2}$, then

$$
M\left(\mathcal{F}^{-1}\left(\rho_{v} \widehat{f}\right)\right)(x) \sim M\left(\mathcal{F}^{-1}\left(\rho_{v} \widehat{f}\right)\right)\left(x^{\prime}\right) .
$$


So, we have

$$
\begin{aligned}
\sum_{(y, v) \in G^{R} \times V_{j}^{R}}\left|h_{(y, v)}\right|^{2} & =R^{n / 2} \sum_{(y, v) \in G^{R} \times V_{j}^{R}}\left|M\left(\mathcal{F}^{-1}\left(\rho_{v} \widehat{f}\right)\right)(y)\right|^{2} \\
& \lesssim \sum_{v \in V_{j}^{R}} \int\left|M\left(\mathcal{F}^{-1}\left(\rho_{v} \widehat{f}\right)\right)(x)\right|^{2} d x .
\end{aligned}
$$

By the Hardy-Littlewood maximal theorem and the Plancherel theorem, we have

$$
\sum_{(y, v) \in G^{R} \times V_{j}^{R}}\left|h_{(y, v)}\right|^{2} \lesssim \sum_{v} \int\left|\rho_{v} \widehat{f}(\xi)\right|^{2} d \xi \lesssim\|f\|_{2}^{2} .
$$

To exploit the spatial localization of the wave packets, we decompose the wave packets further. Let $Q_{R}$ be a cube of side-length $R$ and let $\ell \geq 0$ be an integer. We define $\mathcal{Q}_{\ell}\left(Q_{R}\right)$ as the collection of subcubes which come from partitioning $Q_{R}$ into $2^{(n+1) \ell}$ cubes of side-length $2^{-\ell} R$. For each cube $b \in \mathcal{Q}_{\ell}\left(Q_{R}\right)$, we decompose the wave packets of $U_{j}^{\lambda}$ based on the criterion of whether it meets a cube $b$ or not. We define a bush $U_{j}^{(b), V}=\left(U_{j}^{\lambda}\right)^{(b), V}$ with respect to $V \in L_{l o c}^{2}$ by

$$
U_{j}^{(b), V}=\sum_{T_{j}=(y, v) \in \mathcal{T}_{j}^{R}} \frac{\left\|\tilde{\chi}_{T_{j}} V\right\|_{L^{2}(b)}^{2}}{\left\|\tilde{\chi}_{T_{j}} V\right\|_{L^{2}\left(Q_{R}\right)}^{2}} h_{T_{j}} \phi_{T_{j}},
$$

where $\mathcal{T}_{j}^{R}=G^{R} \times V_{j}^{R}$ and $\tilde{\chi}_{T_{j}}(x, t)=\left(1+\frac{\left|x-\lambda \gamma_{j}^{v}(t / \lambda)\right|}{R^{1 / 2}}\right)^{-N^{10}}$ for $j=1,2$.

If we set

$$
f^{(b), V}=\sum_{T_{j} \in \mathcal{T}_{j}^{R}} \frac{\left\|\tilde{\chi}_{T_{j}} V\right\|_{L^{2}(b)}^{2}}{\left\|\tilde{\chi}_{T_{j}} V\right\|_{L^{2}\left(Q_{R}\right)}^{2}} f_{T_{j}}
$$

then we can write

$$
U_{j}^{(b), V}=\mathcal{G}_{j}^{\lambda} f^{(b), V}
$$

Thus, we are able to decompose $U_{j}^{\lambda}$ as

$$
U_{j}^{\lambda}=\sum_{b \in \mathcal{Q}_{\ell}\left(Q_{R}\right)} U_{j}^{(b), V}
$$

We also define

$$
\left[U_{j}^{V}\right]_{\ell}=\sum_{b \in \mathcal{Q}_{\ell}\left(Q_{R}\right)} \chi_{b} U_{j}^{(b), V},
$$

where $\chi$ denotes a characteristic function.

Proposition 3.2. Let $\lambda \geq R \geq C_{0}$ and $0<c \leq 2^{-C_{0}}$. Let $Q_{R}$ be a cube of side-length $R$. Then for $p=\frac{n+3}{n+1}$, we have

$$
\left\|U_{1}^{\lambda} U_{2}^{\lambda}\right\|_{L^{p}\left(Q_{R}\right)} \leq(1+C N c)\left(\sum_{b \in \mathcal{Q}_{C_{0}}\left(\widetilde{Q}_{R}\right)}\left\|U_{1}^{(b)} U_{2}^{(b)}\right\|_{L^{p}(b)}^{p}\right)^{1 / p}+2^{C C_{0}} c^{-C}
$$


and

$$
\begin{aligned}
& \left\|U_{1}^{\lambda} U_{2}^{\lambda}\right\|_{L^{p}\left(Q_{R} \cap \Lambda^{\lambda}(r)\right)} \\
\leq & (1+C N c)\left(\sum_{b \in \mathcal{Q}_{C_{0}}\left(\widetilde{Q}_{R}\right)}\left\|U_{1}^{(b)} U_{2}^{(b)}\right\|_{L^{p}\left(b \cap \Lambda^{\lambda}(r)\right)}^{p}\right)^{1 / p}+2^{C C_{0}} c^{-C}\left(\frac{R}{r}\right)^{-\frac{n-1}{2(n+3)}} .
\end{aligned}
$$

Here, $\widetilde{Q}_{R}$ is a cube of side-length $C R$ and is contained in $C^{2} Q_{R}$. The $U_{1}^{(b)}, U_{2}^{(b)}$ stand for $U_{1}^{(b), U_{2}}, U_{2}^{(b),\left[U_{1}^{U_{2}}\right]_{C_{0}}}$, respectively.

\section{Proof of the main proposition}

We first state an averaging lemma.

Lemma 4.1 (20, Lemma 6.1). Let $Q$ be a cube of side-length $R \geq C_{0}$ and let $0<c \leq 2^{-C_{0}}$. Then for any $1 \leq p \leq \infty$, there exists a cube $\widetilde{Q}$ of side-length $C R$ which is contained in $C^{2} Q$ such that

$$
\|f\|_{L^{p}(Q)} \leq(1+C N c)\|f\|_{L^{p}\left(X\left(\widetilde{Q}_{R}\right)\right)},
$$

where $X(Q)$ is defined by

$$
X(Q)=\bigcup_{b \in \mathcal{Q}_{C_{0}}(Q)}(1-c) b .
$$

This averaging lemma allows us to replace a cube $Q$ with subcubes which have a nonzero distance between them. In the proof of Proposition 4.2, we use this property to avoid a critical situation.

We begin with reducing the estimates (3.5) and (3.6). By Lemma 4.1 it suffices to show that

$$
\left\|U_{1}^{\lambda} U_{2}^{\lambda}\right\|_{L^{p}\left(X\left(\widetilde{Q}_{R}\right)\right)} \leq\left(\sum_{b \in \mathcal{Q}_{C_{0}}\left(\widetilde{Q}_{R}\right)}\left\|U_{1}^{(b)} U_{2}^{(b)}\right\|_{L^{p}(b)}^{p}\right)^{1 / p}+2^{C C_{0}} c^{-C}
$$

and

$$
\begin{aligned}
\left\|U_{1}^{\lambda} U_{2}^{\lambda}\right\|_{L^{p}\left(X\left(\widetilde{Q}_{R}\right) \cap \Lambda^{\lambda}(r)\right)} \leq & \left(\sum_{b \in \mathcal{Q}_{C_{0}}\left(\widetilde{Q}_{R}\right)}\left\|U_{1}^{(b)} U_{2}^{(b)}\right\|_{L^{p}\left(b \cap \Lambda^{\lambda}(r)\right)}^{p}\right)^{1 / p} \\
& +2^{C C_{0}} c^{-C}\left(\frac{R}{r}\right)^{-\frac{n-1}{2(n+3)}} .
\end{aligned}
$$

By the triangle inequality, we have

$$
\begin{gathered}
\left\|U_{1} U_{2}\right\|_{L^{p}\left(X\left(\widetilde{Q}_{R}\right)\right)} \leq\left\|\left[U_{1}^{U_{2}}\right]_{C_{0}}\left[U_{2}^{\left[U_{1}^{U_{2}}\right]_{C_{0}}}\right]_{C_{0}}\right\|_{L^{p}\left(X\left(\widetilde{Q}_{R}\right)\right)} \\
+\left\|\left(U_{1}-\left[U_{1}^{U_{2}}\right]_{C_{0}}\right) U_{2}\right\|_{L^{p}\left(X\left(\widetilde{Q}_{R}\right)\right)} \\
\quad+\left\|\left(U_{2}-\left[U_{2}^{\left[U_{1}^{U_{2}}\right]_{C_{0}}}\right]_{C_{0}}\right)\left[U_{1}^{U_{2}}\right]_{C_{0}}\right\|_{L^{p}\left(X\left(\widetilde{Q}_{R}\right)\right)},
\end{gathered}
$$

and we also have a similar estimate for $\left\|U_{1} U_{2}\right\|_{L^{p}\left(X\left(\widetilde{Q}_{R}\right) \cap \Lambda(r)\right)}$. 
A simple calculation gives that

$$
\begin{gathered}
\left\|\left[U_{1}^{U_{2}}\right]_{C_{0}}\left[U_{2}^{\left[U_{1}^{U_{2}}\right]_{C_{0}}}\right]_{C_{0}}\right\|_{L^{p}\left(X\left(\widetilde{Q}_{R}\right)\right)} \leq\left(\sum_{b \in \mathcal{Q}_{C_{0}}\left(\widetilde{Q}_{R}\right)}\left\|U_{1}^{(b)} U_{2}^{(b)}\right\|_{L^{p}(b)}^{p}\right)^{1 / p}, \\
\left\|\left[U_{1}^{U_{2}}\right]_{C_{0}}\left[U_{2}^{\left[U_{1}^{U_{2}}\right]_{C_{0}}}\right]_{C_{0}}\right\|_{L^{p}\left(X\left(\widetilde{Q}_{R}\right) \cap \Lambda(r)\right)} \leq\left(\sum_{b \in \mathcal{Q}_{C_{0}}\left(\widetilde{Q}_{R}\right)}\left\|U_{1}^{(b)} U_{2}^{(b)}\right\|_{L^{p}(b \cap \Lambda(r))}^{p}\right)^{1 / p} .
\end{gathered}
$$

Now, we have to show that

$$
\begin{aligned}
\left\|\left(U_{1}-\left[U_{1}^{U_{2}}\right]_{C_{0}}\right) U_{2}\right\|_{L^{p}\left(X\left(\widetilde{Q}_{R}\right)\right)}+\left\|\left(U_{2}-\left[U_{2}^{\left[U_{1}^{U_{2}}\right]_{C_{0}}}\right]_{C_{0}}\right)\left[U_{1}^{U_{2}}\right]_{C_{0}}\right\|_{L^{p}\left(X\left(\widetilde{Q}_{R}\right)\right)} & \lesssim 2^{C C_{0}} c^{-C}
\end{aligned}
$$

and

$$
\begin{aligned}
\left\|\left(U_{1}-\left[U_{1}^{U_{2}}\right]_{C_{0}}\right) U_{2}\right\|_{L^{p}\left(X\left(\widetilde{Q}_{R}\right) \cap \Lambda(r)\right)}+\left\|\left(U_{2}-\left[U_{2}^{\left[U_{1}^{U_{2}}\right]_{C_{0}}}\right]_{C_{0}}\right)\left[U_{1}^{U_{2}}\right]_{C_{0}}\right\|_{L^{p}\left(X\left(\widetilde{Q}_{R}\right) \cap \Lambda(r)\right)} \\
\lesssim 2^{C C_{0}} c^{-C}\left(\frac{R}{r}\right)^{-\frac{n-1}{2(n+3)}}
\end{aligned}
$$

Since the proofs of the estimates for

$$
\left(U_{1}-\left[U_{1}^{U_{2}}\right]_{C_{0}}\right) U_{2} \text { and }\left(U_{2}-\left[U_{2}^{\left[U_{1}^{U_{2}}\right]_{C_{0}}}\right]_{C_{0}}\right)\left[U_{1}^{U_{2}}\right]_{C_{0}}
$$

are almost the same, we only need to prove that

$$
\begin{aligned}
\left\|\left(U_{1}-\left[U_{1}^{U_{2}}\right]_{C_{0}}\right) U_{2}\right\|_{L^{p}\left(X\left(\widetilde{Q}_{R}\right)\right)} & \lesssim 2^{C C_{0}} c^{-C} \\
\left\|\left(U_{1}-\left[U_{1}^{U_{2}}\right]_{C_{0}}\right) U_{2}\right\|_{L^{p}\left(X\left(\widetilde{Q}_{R}\right) \cap \Lambda(r)\right)} & \lesssim 2^{C C_{0}} c^{-C}\left(\frac{R}{r}\right)^{-\frac{n-1}{2(n+3)}} .
\end{aligned}
$$

By interpolation it suffices to show that

$$
\begin{aligned}
\left\|\left(U_{1}-\left[U_{1}^{U_{2}}\right]_{C_{0}}\right) U_{2}\right\|_{L^{2}\left(X\left(\widetilde{Q}_{R}\right)\right)} & \lesssim 2^{C C_{0}} c^{-C} R^{(1-n) / 4}, \\
\left\|\left(U_{1}-\left[U_{1}^{U_{2}}\right]_{C_{0}}\right) U_{2}\right\|_{L^{2}\left(X\left(\widetilde{Q}_{R}\right) \cap \Lambda(r)\right)} & \lesssim 2^{C C_{0}} c^{-C} R^{(1-n) / 4},
\end{aligned}
$$

and

$$
\begin{aligned}
\left\|\left(U_{1}-\left[U_{1}^{U_{2}}\right]_{C_{0}}\right) U_{2}\right\|_{L^{1}\left(X\left(\widetilde{Q}_{R}\right)\right)} & \lesssim c^{-C} R \\
\left\|\left(U_{1}-\left[U_{1}^{U_{2}}\right]_{C_{0}}\right) U_{2}\right\|_{L^{1}\left(X\left(\widetilde{Q}_{R}\right) \cap \Lambda(r)\right)} & \lesssim c^{-C} r^{1 / 2} R^{1 / 2},
\end{aligned}
$$

respectively.

4.1. The $\left(L^{2} \times L^{2} \rightarrow L^{2}\right)$-bilinear estimate.

Proposition 4.2. Let $\lambda \geq R \geq C_{0}$ and $0<c \leq 2^{-C_{0}}$. Suppose that $Q$ is a cube of side-length $C R$. Then we have

$$
\begin{gathered}
\left\|\left(U_{1}-\left[U_{1}^{U_{2}}\right]_{C_{0}}\right) U_{2}\right\|_{L^{2}(X(Q))} \lesssim 2^{C C_{0}} c^{-C} R^{(1-n) / 4}, \\
\left\|\left(U_{1}-\left[U_{1}^{U_{2}}\right]_{C_{0}}\right) U_{2}\right\|_{L^{2}(X(Q) \cap \Lambda(r))} \lesssim 2^{C C_{0}} c^{-C} R^{(1-n) / 4} .
\end{gathered}
$$

Proof. The last estimate is trivial because

$$
\left\|\left(U_{1}-\left[U_{1}^{U_{2}}\right]_{C_{0}}\right) U_{2}\right\|_{L^{2}(X(Q) \cap \Lambda(r))} \leq\left\|\left(U_{1}-\left[U_{1}^{U_{2}}\right]_{C_{0}}\right) U_{2}\right\|_{L^{2}(X(Q))} .
$$

Consider the first estimate. By (3.4) and the triangle inequality, we have

$$
\left\|\left(U_{1}-\left[U_{1}^{U_{2}}\right]_{C_{0}}\right) U_{2}\right\|_{L^{2}(X(Q))} \lesssim \sum_{b \in \mathcal{Q}_{C_{0}}(Q)}\left\|U_{1}^{(b)} U_{2}\right\|_{L^{2}(X(Q) \backslash b)},
$$


where $U_{1}^{(b)}=U_{1}^{(b), U_{2}}$. Since the number of cubes in $\mathcal{Q}_{C_{0}}(Q)$ is fixed, namely exactly $2^{(n+1) C_{0}}$, it suffices to show that

$$
\left\|U_{1}^{(b)} U_{2}\right\|_{L^{2}(X(Q) \backslash b)} \lesssim c^{-C} R^{(1-n) / 4} .
$$

To use the spatial localization of the wave packets, we further decompose $Q$ into $R^{1 / 2}$-scale cubes. Let $\kappa$ be a positive integer with $2^{-\kappa} R \sim R^{1 / 2}$. Then the square of (4.1) is such that

$$
\sum_{q \in \mathcal{Q}_{\kappa}(Q): \operatorname{dist}(q, b) \gtrsim c R}\left\|U_{1}^{(b)} U_{2}\right\|_{L^{2}(q)}^{2} \lesssim c^{-C} R^{(1-n) / 2} .
$$

Here, the condition that $\operatorname{dist}(q, b) \gtrsim c R$ follows from the definition of $X(Q)$. By (3.2) and the triangle inequality, the summand has the bound

$$
\left\|U_{1}^{(b)} U_{2}\right\|_{L^{2}(q)} \lesssim \sum_{T_{1} \in \mathcal{T}_{1}^{R}} \frac{\left\|\tilde{\chi}_{T_{1}} U_{2}\right\|_{L^{2}(b)}^{2}}{\left\|\tilde{\chi}_{T_{1}} U_{2}\right\|_{L^{2}(Q)}^{2}} h_{T_{1}}\left\|\phi_{T_{1}} U_{2}\right\|_{L^{2}(q)} .
$$

Let $z_{q}$ denote the center of the cube $q$. By the Cauchy-Schwarz inequality and the fact that

$$
\frac{\left\|\tilde{\chi}_{T_{1}} U_{2}\right\|_{L^{2}(b)}^{2}}{\left\|\tilde{\chi}_{T_{1}} U_{2}\right\|_{L^{2}(Q)}^{2}} \leq \frac{\left\|\tilde{\chi}_{T_{1}} U_{2}\right\|_{L^{2}(b)}}{\left\|\tilde{\chi}_{T_{1}} U_{2}\right\|_{L^{2}(Q)}}
$$

we have

$$
\left\|U_{1}^{(b)} U_{2}\right\|_{L^{2}(q)}^{2} \lesssim\left(\sum_{T_{1} \in \mathcal{T}_{1}^{R}} \frac{h_{T_{1}}^{2}\left\|\phi_{T_{1}} U_{2}\right\|_{L^{2}(q)}^{2}}{\tilde{\chi}_{T_{1}}\left(z_{q}\right)^{2}\left\|\tilde{\chi}_{T_{1}} U_{2}\right\|_{L^{2}(Q)}^{2}}\right)\left(\sum_{T_{1} \in \mathcal{T}_{1}^{R}}\left\|\tilde{\chi}_{T_{1}} U_{2}\right\|_{L^{2}(b)}^{2} \tilde{\chi}_{T_{1}}\left(z_{q}\right)^{2}\right) .
$$

By summation we get

$$
\sum_{T_{1} \in \mathcal{T}_{1}^{R}}\left\|\tilde{\chi}_{T_{1}} U_{2}\right\|_{L^{2}(b)}^{2} \tilde{\chi}_{T_{1}}\left(z_{q}\right)^{2} \lesssim\left\|U_{2} \mathcal{V}\right\|_{2}^{2}
$$

where

$$
\mathcal{V}=\left(\sum_{T_{1} \in \mathcal{T}_{1}^{R}} \tilde{\chi}_{T_{1}}\left(z_{q}\right)^{2} \tilde{\chi}_{T_{1}}^{2}\right)^{1 / 2} \chi_{b}
$$

Since $\operatorname{dist}(q, b) \gtrsim c R$, we have

$$
|\mathcal{V}(z)| \lesssim c^{-C}\left(1+\frac{\operatorname{dist}\left(z, \Lambda_{1}^{\lambda, z_{q}}\right)}{R^{1 / 2}}\right)^{-N}
$$

By Proposition 2.2 we obtain

$$
\left\|U_{2} \mathcal{V}\right\|_{2}^{2} \lesssim c^{-C} R^{1 / 2} .
$$

To prove (4.2), it now remains to show that

$$
\sum_{\substack{q \in \mathcal{Q}_{\kappa}(Q) \\: \operatorname{dist}(q, b) \gtrsim c R}} \sum_{T_{1} \in \mathcal{T}_{1}^{R}} \frac{h_{T_{1}}^{2}\left\|\phi_{T_{1}} U_{2}\right\|_{L^{2}(q)}^{2}}{\tilde{\chi}_{T_{1}}\left(z_{q}\right)^{2}\left\|\tilde{\chi}_{T_{1}} U_{2}\right\|_{L^{2}(Q)}^{2}} \lesssim c^{-C} R^{-n / 2} .
$$

Since

$$
\frac{\left\|\phi_{T_{1}} U_{2}\right\|_{L^{2}(q)}^{2}}{\tilde{\chi}_{T_{1}}\left(z_{q}\right)^{2}} \lesssim\left\|\phi_{T_{1}} U_{2}\right\|_{L^{2}(q)}^{2},
$$


by (11) in Lemma 3.1, we have

$$
\sum_{\substack{q \in \mathcal{Q}_{\kappa}(Q) ; \\ \operatorname{dist}(q, b) \gtrsim c R}} \frac{\left\|\phi_{T_{1}} U_{2}\right\|_{L^{2}(q)}^{2}}{\tilde{\chi}_{T_{1}}\left(z_{q}\right)^{2}\left\|\tilde{\chi}_{T_{1}} U_{2}\right\|_{L^{2}(Q)}^{2}} \lesssim \sum_{q \in \mathcal{Q}_{\kappa}(Q)} \frac{\left\|\phi_{T_{1}} U_{2}\right\|_{L^{2}(q)}^{2}}{\left\|\tilde{\chi}_{T_{1}} U_{2}\right\|_{L^{2}(Q)}^{2}} \lesssim c^{-C} R^{-n / 2} .
$$

Thus, by (2) in Lemma 3.1, we obtain (4.3).

4.2. The $\left(L^{2} \times L^{2} \rightarrow L^{1}\right)$-bilinear estimate.

Proposition 4.3. Let $\lambda \geq R \geq C_{0}, 0<c<2^{-C_{0}}$ and let $Q$ be a cube of side-length $R$. Then we have

$$
\begin{gathered}
\left\|\left(U_{1}-\left[U_{1}^{U_{2}}\right]_{C_{0}}\right) U_{2}\right\|_{L^{1}(X(Q))} \lesssim c^{-C} R, \\
\left\|\left(U_{1}-\left[U_{1}^{U_{2}}\right]_{C_{0}}\right) U_{2}\right\|_{L^{1}(X(Q) \cap \Lambda(r))} \lesssim c^{-C} R^{1 / 2} r^{1 / 2} .
\end{gathered}
$$

Proof. Let us now consider (4.4). We have $X(Q) \subset Q$. Using Hölder's inequality and the triangle inequality, we get

$$
\begin{aligned}
\left\|\left(U_{1}-\left[U_{1}^{U_{2}}\right]_{C_{0}}\right) U_{2}\right\|_{L^{1}(Q)} & \leq\left\|U_{1}-\left[U_{1}^{U_{2}}\right]_{C_{0}}\right\|_{L^{2}(Q)}\left\|U_{2}\right\|_{L^{2}(Q)} \\
& \leq\left(\left\|U_{1}\right\|_{L^{2}(Q)}+\left\|\left[U_{1}^{U_{2}}\right]_{C_{0}}\right\|_{L^{2}(Q)}\right)\left\|U_{2}\right\|_{L^{2}(Q)} .
\end{aligned}
$$

By the generalized Plancherel theorem, we have

$$
\left\|U_{1}\right\|_{L^{2}(Q)} \lesssim R^{1 / 2}\|f\|_{2} \quad \text { and } \quad\left\|U_{2}\right\|_{L^{2}(Q)} \lesssim R^{1 / 2}\|g\|_{2}
$$

Similarly, we have

$$
\left\|\left[U_{1}^{U_{2}}\right]_{C_{0}}\right\|_{L^{2}(Q)} \lesssim 2^{-C_{0} / 2} R^{1 / 2}\|f\|_{2} .
$$

Indeed, if $b \in \mathcal{Q}_{C_{0}}(Q)$, by the generalized Plancherel theorem we have

$$
\left\|U_{1}^{(b)}\right\|_{L^{2}(b)} \lesssim 2^{-C_{0} / 2} R^{1 / 2}\left\|f^{(b)}\right\|_{2}
$$

where $f^{(b)}=\sum_{T \in \mathcal{T}_{1}} m_{T}^{(b)} \eta_{y} \mathcal{F}^{-1}\left(\rho_{v} \widehat{f}\right)$ with $m_{T}^{(b)}=\left\|\tilde{\chi}_{T} U_{2}\right\|_{L^{2}(b)}^{2} /\left\|\tilde{\chi}_{T} U_{2}\right\|_{L^{2}(Q)}^{2}$. Thus, by orthogonality and Plancherel's theorem, we have

$$
\begin{aligned}
\left\|\left[U_{1}^{U_{2}}\right]_{C_{0}}\right\|_{L^{2}(Q)}^{2} & =\sum_{b \in \mathcal{Q}_{C_{0}}(Q)}\left\|U_{1}^{(b)}\right\|_{L^{2}(b)}^{2} \\
& \lesssim 2^{-C_{0}} R \sum_{b \in \mathcal{Q}_{C_{0}}(Q)}\left\|f^{(b)}\right\|_{2}^{2} \\
& \lesssim 2^{-C_{0}} R\|f\|_{2}^{2} .
\end{aligned}
$$

Combining (4.6) and (4.7), we obtain (4.4).

Now, consider (4.5). We have

$$
\left\|\left(U_{1}-\left[U_{1}^{U_{2}}\right]_{C_{0}}\right) U_{2}\right\|_{L^{1}(Q \cap \Lambda(r))} \leq \sum_{j=1,2}\left\|\left(U_{1}-\left[U_{1}^{U_{2}}\right]_{C_{0}}\right) U_{2}\right\|_{L^{1}\left(Q \cap \Lambda_{j}^{\lambda}(r)\right)} .
$$

By Hölder's inequality and the triangle inequality, we have

$$
\begin{aligned}
\|\left(U_{1}-\left[U_{1}^{U_{2}}\right]_{C_{0}}\right) & U_{2} \|_{L^{1}\left(Q \cap \Lambda_{j}^{\lambda}(r)\right)} \\
& \leq\left\|U_{1}-\left[U_{1}^{U_{2}}\right]_{C_{0}}\right\|_{L^{2}\left(Q \cap \Lambda_{j}^{\lambda}(r)\right)}\left\|U_{2}\right\|_{L^{2}\left(Q \cap \Lambda_{j}^{\lambda}(r)\right)} \\
& \leq\left(\left\|U_{1}\right\|_{L^{2}\left(Q \cap \Lambda_{j}^{\lambda}(r)\right)}-\left\|\left[U_{1}^{U_{2}}\right]_{C_{0}}\right\|_{L^{2}\left(Q \cap \Lambda_{j}^{\lambda}(r)\right)}\right)\left\|U_{2}\right\|_{L^{2}\left(Q \cap \Lambda_{j}^{\lambda}(r)\right)} .
\end{aligned}
$$


By Proposition 2.2, we get

$$
\begin{aligned}
\left\|U_{1}\right\|_{L^{2}\left(Q \cap \Lambda_{2}(r)\right)} & \lesssim r^{1 / 2}\|f\|_{2}, \\
\left\|U_{2}\right\|_{L^{2}\left(Q \cap \Lambda_{1}(r)\right)} & \lesssim r^{1 / 2}\|g\|_{2}, \\
\left\|\left[U_{1}^{U_{2}}\right]_{C_{0}}\right\|_{L^{2}\left(Q \cap \Lambda_{2}(r)\right)} & \lesssim 2^{-C_{0} / 2} r^{1 / 2}\|f\|_{2}, \\
\left\|\left[U_{2}^{U_{1}}\right]_{C_{0}}\right\|_{L^{2}\left(Q \cap \Lambda_{1}(r)\right)} & \lesssim 2^{-C_{0} / 2} r^{1 / 2}\|g\|_{2} .
\end{aligned}
$$

By the generalized Plancherel theorem again, we have

$$
\begin{aligned}
\left\|U_{j}\right\|_{L^{2}\left(Q \cap \Lambda_{j}(r)\right)} & \lesssim R^{1 / 2}\|f\|_{2} \\
\left\|\left[U_{j}^{U_{k}}\right]_{C_{0}}\right\|_{L^{2}\left(Q \cap \Lambda_{j}(r)\right)} & \lesssim 2^{-C_{0} / 2} R^{1 / 2}\|f\|_{2},
\end{aligned}
$$

for $j, k=1,2$ and $j \neq k$. Combining these, we obtain (4.5).

\section{RECURSive ineQuality FOR a SCALE}

In this section, we will utilize the induction-on-scale argument. The following definition of $A(\lambda)$ will play the role of an induction hypothesis for us:

Hypothesis 5.1. Let $\lambda \geq C_{0}$. Define $A(\lambda)$ to be the least constant such that the inequality

$$
\left\|U_{1}^{\lambda} U_{2}^{\lambda}\right\|_{p} \leq A(\lambda) E\left(U_{1}^{\lambda}\right)^{1 / 2} E\left(U_{2}^{\lambda}\right)^{1 / 2}
$$

holds for all $\mathcal{O}(1)$ smooth perturbations of the phases $\Psi_{1}$ and $\Psi_{2}$.

In this section, we define the energy as follows:

$$
E\left(\mathcal{G}_{j}^{\lambda} f\right)=\|f\|_{2}^{2} .
$$

By (1.11), we have $E\left(U_{1}^{\lambda}\right)=E\left(U_{2}^{\lambda}\right)=1$.

Under this induction hypothesis, we will show the following recursive inequality.

Proposition 5.2. If $\lambda \geq C_{0}$ and $0<c \leq 2^{-C_{0}}$, then we have

$$
A(\lambda) \leq(1+C N c) \sup _{C_{0} \leq \lambda^{\prime} \leq \lambda / 2} A\left(\lambda^{\prime}\right)+2^{C C_{0}} c^{-C} .
$$

From this proposition, we can obtain the $\lambda^{\varepsilon}$-loss version of Theorem 1.1 Indeed, let us define

$$
\overline{A(\lambda)}=\sup _{C_{0} \leq R \leq \lambda} A(R) .
$$

From (5.2), we have

$$
\overline{A(\lambda)} \leq(1+C N c) \overline{A(\lambda / 2)}+2^{C C_{0}} c^{-C} .
$$

Setting $c=\lambda^{-\frac{\varepsilon}{C N}}$, we iterate the recursive inequality and use the crude estimate $\left\|U_{1}^{\lambda} U_{2}^{\lambda}\right\|_{p} \lesssim \lambda^{C}$. Then we obtain

$$
A(\lambda) \lesssim 2^{C C_{0}} \lambda^{\varepsilon / N}
$$

for any $\varepsilon>0$.

Proposition 5.2 is obtained by applying the induction argument to (3.5) in Proposition 3.2 To do that, we need the following lemma. 
Lemma 5.3. Let $b$ be $a$ cube of side-length $\ell$ with center $z_{b}=\left(x_{b}, t_{b}\right)$. Then we have

$$
\left\|\mathcal{G}_{1}^{\lambda} f \mathcal{G}_{2}^{\lambda} g\right\|_{L^{p}(b)}=\left\|\widetilde{\mathcal{G}}_{1}^{\ell} \tilde{f} \widetilde{\mathcal{G}}_{2}^{\ell} \tilde{g}\right\|_{p}
$$

where $\tilde{f}, \tilde{g}$ are some functions such that $|f|=|\tilde{f}|,|g|=|\tilde{g}|$, and the phase of $\widetilde{\mathcal{G}}_{j}^{\ell}$ is an $\mathcal{O}(\ell / \lambda)$-perturbation of the phase of $\mathcal{G}_{j}^{\ell}$.

Proof. Let

$$
\widetilde{\Psi}_{j}(z, \xi)=\frac{\lambda}{\ell} \Psi_{j}\left(\frac{\ell}{\lambda} z+\frac{z_{b}}{\lambda}, \xi\right)-2^{C_{0}} \Psi_{j}\left(\frac{z_{b}}{\lambda}, \xi\right) .
$$

By Taylor's theorem, we have

$$
\widetilde{\Psi}_{j}(z, \xi)=\left\langle\nabla_{z} \Psi_{j}\left(z_{b} / \lambda, \xi\right), z\right\rangle+\mathcal{O}(\ell / \lambda) .
$$

We can easily check that $\left\langle\nabla_{z} \Psi_{j}\left(z_{b} / \lambda, \xi\right), z\right\rangle$ satisfies the cinematic curvature condition and the separation condition (1.5). Let us define an operator $\widetilde{\mathcal{G}}_{j}^{\varrho} f$ by

$$
\widetilde{\mathcal{G}}_{j}^{\varrho} f(z)=\int e^{i \varrho \widetilde{\Psi}_{j}\left(\varrho^{-1} z, \xi\right)} \widehat{f}(\xi) \widetilde{a}_{j}\left(\varrho^{-1} z, \xi\right) d \xi,
$$

where $\widetilde{a}_{j}(z, \xi)=\chi_{Q_{1}}(z) a_{j}\left(\frac{\ell}{\lambda} z+\frac{z_{b}}{\lambda}, \xi\right)$.

If we take a function $\tilde{f}$ such that

$$
\widehat{\tilde{f}}(\xi)=e^{i \lambda \Psi_{j}\left(z_{b} / \lambda, \xi\right)} \widehat{f}(\xi),
$$

then we have

$$
\widetilde{\mathcal{G}}_{j}^{\ell} \tilde{f}(z)=\chi_{Q_{1}}\left(\ell^{-1} z\right) \mathcal{G}_{j}^{\lambda} f\left(z+z_{b}\right) .
$$

So, we obtain

$$
\left\|\mathcal{G}_{1}^{\lambda} f \mathcal{G}_{2}^{\lambda} g\right\|_{L^{p}(b)}=\left\|\widetilde{\mathcal{G}}_{1}^{\ell} \tilde{f} \widetilde{\mathcal{G}}_{2}^{\ell} \tilde{g}\right\|_{p} .
$$

Lemma 5.4. Let $V_{j}^{R}\left(\xi_{0}\right):=\Xi_{j} \cap\left(R^{-1 / 2} \mathbb{Z}^{n}+\xi_{0}\right)$, where $R^{-1 / 2} \mathbb{Z}^{n}+\xi_{0}$ denotes the $\xi_{0}$-translation of $R^{-1 / 2} \mathbb{Z}^{n}$. Let $q_{0}$ be the cube of side-length $C R^{-1 / 2}$ and centered at the origin. Suppose that $U_{j}^{(b)}$ is defined as (3.2) but with $\mathcal{T}_{j}^{R}=G^{R} \times V_{j}^{R}\left(\xi_{0}\right)$. Then for some $\xi_{0} \in q_{0}$, we have

$$
\left(\sum_{b \in \mathcal{Q}_{C_{0}}\left(\widetilde{Q}_{R}\right)} E\left(U_{j}^{(b)}\right)\right)^{1 / 2} \leq(1+C c) E\left(U_{j}^{\lambda}\right)^{1 / 2} .
$$

Proof. Using (5.1), (3.3) and (3.1) we expand

$$
\begin{aligned}
\left(\sum_{b \in \mathcal{Q}_{C_{0}}\left(\widetilde{Q}_{R}\right)} E\left(U_{j}^{(b)}\right)\right)^{1 / 2} & =\left(\sum_{b \in \mathcal{Q}_{C_{0}}\left(\widetilde{Q}_{R}\right)}\left\|\sum_{T_{j} \in \mathcal{T}_{j}^{R}} m_{T_{j}}^{(b)} f_{T_{j}}\right\|_{2}^{2}\right)^{1 / 2} \\
& =\left(\sum_{b \in \mathcal{Q}_{C_{0}}\left(\widetilde{Q}_{R}\right)}\left\|\sum_{v \in V_{j}^{R}\left(\xi_{0}\right)} \sum_{y \in G^{R}} m_{(y, v)}^{(b)}\left(\eta_{y} \mathcal{F}^{-1}\left(\rho_{v} \widehat{f}\right)\right)\right\|_{2}^{2}\right)^{1 / 2}
\end{aligned}
$$

where $m_{T_{j}}^{(b)}=\left\|\tilde{\chi}_{T_{j}} V\right\|_{L^{2}(b)}^{2} /\left\|\tilde{\chi}_{T_{j}} V\right\|_{L^{2}\left(\widetilde{Q}_{R}\right)}^{2}$. Note that

$$
\sum_{b \in \mathcal{Q}_{C_{0}}\left(\widetilde{Q}_{R}\right)} m_{T_{j}}^{(b)}=1
$$


To obtain the factor $(1+C c)$, we need more careful analysis for the frequency decomposition. Let $W_{j}^{R}\left(\xi_{0}\right) \subset \Xi_{j}$ be a set defined by

$$
W_{j}^{R}\left(\xi_{0}\right):=\bigcup_{v \in V_{j}^{R}\left(\xi_{0}\right)}\left\{\xi \in \Xi_{j}:|\xi-v|<\left(1-C c^{2}\right) R^{-1 / 2}\right\} .
$$

By the triangle inequality, we have

$$
\begin{aligned}
& \left(\sum_{b \in \mathcal{Q}_{C_{0}}\left(\widetilde{Q}_{R}\right)} E\left(U_{j}^{(b)}\right)\right)^{1 / 2} \\
& \quad \leq\left(\sum_{b \in \mathcal{Q}_{C_{0}}\left(\widetilde{Q}_{R}\right)}\left\|\sum_{v \in V_{j}^{R}\left(\xi_{0}\right)} \sum_{y \in G^{R}} m_{(y, v)}^{(b)}\left(\eta_{y} \mathcal{F}^{-1}\left(\chi_{W_{j}^{R}\left(\xi_{0}\right)} \rho_{v} \widehat{f}\right)\right)\right\|_{2}^{2}\right)^{1 / 2} \\
& +\left(\sum_{b \in \mathcal{Q}_{C_{0}}\left(\widetilde{Q}_{R}\right)}\left\|\sum_{v \in V_{j}^{R}\left(\xi_{0}\right)} \sum_{y \in G^{R}} m_{(y, v)}^{(b)}\left(\eta_{y} \mathcal{F}^{-1}\left(\left(1-\chi_{W_{j}^{R}\left(\xi_{0}\right)}\right) \rho_{v} \widehat{f}\right)\right)\right\|_{2}^{2}\right)^{1 / 2} \\
& =:(\mathrm{I})+(\mathrm{II}) .
\end{aligned}
$$

First, consider (I). By orthogonality we have

$$
(\mathrm{I})=\left(\sum_{b \in \mathcal{Q}_{C_{0}}\left(\widetilde{Q}_{R}\right)} \sum_{v \in V_{j}^{R}\left(\xi_{0}\right)}\left\|\sum_{y \in G^{R}} m_{(y, v)}^{(b)}\left(\eta_{y} \mathcal{F}^{-1}\left(\chi_{W_{j}^{R}\left(\xi_{0}\right)} \rho_{v} \widehat{f}\right)\right)\right\|_{2}^{2}\right)^{1 / 2} .
$$

By rearranging, we have

$$
\left(\sum_{v \in V_{j}^{R}\left(\xi_{0}\right)} \int \sum_{b \in \mathcal{Q}_{C_{0}}\left(\widetilde{Q}_{R}\right)}\left|\sum_{y \in G^{R}} m_{(y, v)}^{(b)} \eta_{y}(x)\right|^{2}\left|\mathcal{F}^{-1}\left(\chi_{W_{j}^{R}\left(\xi_{0}\right)} \rho_{v} \widehat{f}\right)(x)\right|^{2} d x\right)^{1 / 2} .
$$

Since

$$
\sum_{b \in \mathcal{Q}_{C_{0}}\left(\widetilde{Q}_{R}\right)}\left|\sum_{y \in G^{R}} m_{(y, v)}^{(b)} \eta_{y}(x)\right|^{2} \leq\left|\sum_{b \in \mathcal{Q}_{C_{0}}\left(\widetilde{Q}_{R}\right)} \sum_{y \in G^{R}} m_{(y, v)}^{(b)} \eta_{y}(x)\right|^{2},
$$

by (5.3) we have

$$
(\mathrm{I}) \leq\left(\sum_{v \in V_{j}^{R}\left(\xi_{0}\right)} \int\left|\mathcal{F}^{-1}\left(\chi_{W_{j}^{R}\left(\xi_{0}\right)} \rho_{v} \widehat{f}\right)(x)\right|^{2} d x\right)^{1 / 2} .
$$

By orthogonality again, we obtain

$$
(\mathrm{I}) \leq\left(\int\left|\mathcal{F}^{-1}\left(\chi_{W_{j}^{R}\left(\xi_{0}\right)} \widehat{f}\right)(x)\right|^{2} d x\right)^{1 / 2} \leq\|f\|_{2}=E\left(U_{j}^{\lambda}\right)^{1 / 2} .
$$

Now consider (II). In a similar manner, but using almost orthogonality instead of orthogonality we have

$$
(\mathrm{II}) \lesssim\left(\int\left|\mathcal{F}^{-1}\left(\left(1-\chi_{W_{j}^{R}\left(\xi_{0}\right)}\right) \widehat{f}\right)(x)\right|^{2} d x\right)^{1 / 2}=\left\|\left(1-\chi_{W_{j}^{R}\left(\xi_{0}\right)}\right) \widehat{f}\right\|_{2} .
$$

It suffices to show that for some $\xi_{0} \in q_{0}$, we have

$$
\left\|\left(1-\chi_{W_{j}^{R}\left(\xi_{0}\right)}\right) \widehat{f}\right\|_{2} \lesssim c\|f\|_{2} .
$$


We may restrict the support of $\widehat{f}$ to $\Xi_{j}$. By the pigeonhole principle and the Plancherel theorem, it suffices to show that

$$
\frac{1}{\left|q_{0}\right|} \int_{q_{0}}\left\|\left(1-\chi_{W_{j}^{R}\left(\xi_{0}\right)}\right) f\right\|_{L^{2}\left(\Xi_{j}\right)}^{2} d \xi_{0} \lesssim c^{2}\|f\|_{2}^{2}
$$

The left side of (5.6) is written as

$$
\begin{aligned}
& \frac{1}{\left|q_{0}\right|} \int_{q_{0}} \int_{\Xi_{j}}\left(1-\chi_{W_{j}^{R}\left(\xi_{0}\right)}\right)(x)|f(x)|^{2} d x d \xi_{0} \\
& =\frac{1}{\left|q_{0}\right|} \int_{q_{0}} \int_{\Xi_{j}}\left(1-\chi_{W_{j}^{R}(0)}\right)\left(x-\xi_{0}\right)|f(x)|^{2} d x d \xi_{0} \\
& \quad=\frac{1}{\left|q_{0}\right|} \int_{\Xi_{j}}\left(\int_{q_{0}}\left(1-\chi_{W_{j}^{R}(-x)}\right)\left(\xi_{0}\right) d \xi_{0}\right)|f(x)|^{2} d x .
\end{aligned}
$$

Since the inner integral is $\lesssim c^{2}\left|q_{0}\right|$, we obtain (5.6).

Proof of Proposition 5.2. There is a cube $Q$ which contains the $z$-support of $a_{j}$. Let $Q_{\lambda}$ be the $\lambda$-dilation of $Q$. By (3.5) in Proposition 3.2. we have

$$
A(\lambda) \leq(1+C N c)\left(\sum_{b \in \mathcal{Q}_{C_{0}}\left(\widetilde{Q}_{\lambda}\right)}\left\|U_{1}^{(b)} U_{2}^{(b)}\right\|_{L^{p}(b)}^{p}\right)^{1 / p}+2^{C C_{0}} c^{-C} .
$$

By Lemma 5.3 and Hypothesis 5.1, we have

$$
\left\|U_{1}^{(b)} U_{2}^{(b)}\right\|_{L^{p}(b)} \leq A\left(2^{-C_{0}} \lambda\right) E\left(U_{1}^{(b)}\right)^{1 / 2} E\left(U_{2}^{(b)}\right)^{1 / 2} .
$$

So, $A(\lambda)$ is bounded by

$$
(1+C N c) \sup _{C_{0} \leq \lambda^{\prime} \leq \lambda / 2} A\left(\lambda^{\prime}\right)\left(\sum_{b \in \mathcal{Q}_{C_{0}}\left(\widetilde{Q}_{\lambda}\right)} E\left(U_{1}^{(b)}\right)^{\frac{p}{2}} E\left(U_{2}^{(b)}\right)^{\frac{p}{2}}\right)^{1 / p}+2^{C C_{0}} c^{-C} .
$$

Since $p \geq 1$, we have

$$
\left(\sum_{b \in \mathcal{Q}_{C_{0}}\left(\widetilde{Q}_{\lambda}\right)} E\left(U_{1}^{(b)}\right)^{\frac{p}{2}} E\left(U_{2}^{(b)}\right)^{\frac{p}{2}}\right)^{1 / p} \leq \sum_{b \in \mathcal{Q}_{C_{0}}\left(\widetilde{Q}_{\lambda}\right)} E\left(U_{1}^{(b)}\right)^{\frac{1}{2}} E\left(U_{2}^{(b)}\right)^{\frac{1}{2}}
$$

Apply the Cauchy-Schwarz inequality and Lemma 5.4 to the right side of the above estimate. Then it is bounded by $(1+C c)^{2}$. Since $c$ is small enough, we obtain (5.2).

\section{Spatial LOCALization}

In this section, we develop some machinery to deal with the spatial localization. We first take a sequence $\left\{f_{\alpha}\right\}$ that converges to a given $f$ in $L^{2}$ as $\alpha \rightarrow \infty$, which makes it easy to localize $\mathcal{G}_{j}^{\lambda} f$ to a disc.

We may assume that

$$
a_{j}(x, t, \xi)=a_{\times}(x) a_{\dagger}(t) a_{\Xi_{j}}(\xi)
$$

where $a_{\times}, a_{\dagger}$ and $a_{\Xi_{j}}$ are proper bump functions with compact support, and we have $\int a_{\times}=1$. We denote by $a_{\times}^{\lambda}(x)=a_{\times}(x / \lambda)$.

For a fixed $t_{0} \in \mathbb{R}$ and $\alpha \geq 1$, we define a sequence $f_{\alpha}=f_{\left(t_{0} ; \alpha\right)}$ as

$$
\widehat{f}_{\alpha}(\xi)=\int I_{j}^{\lambda, \alpha}(\zeta, \xi) \widehat{f}(\zeta) d \zeta
$$


where

$$
I_{j}^{\lambda, \alpha}(\zeta, \xi)=I_{j}^{\lambda,\left(t_{0} ; \alpha\right)}(\zeta, \xi)=\int e^{i\left(\Psi_{j}^{\lambda}\left(y, t_{0}, \zeta\right)-\Psi_{j}^{\lambda}\left(y, t_{0}, \xi\right)\right)} a_{\times}^{\lambda}\left(\alpha^{-1} y\right) d y .
$$

Lemma 6.1. Let $f \in L^{2}\left(\mathbb{R}^{n}\right)$. Suppose that the phase $\Psi_{j}$ satisfies the nondegenerate condition (1.2) on $\mathbb{R}^{n} \times\left\{t_{0}\right\} \times \Xi_{j}$. Then $\widehat{f}_{\alpha} \rightarrow \widehat{f}$ a.e. and $f_{\alpha} \rightarrow f$ in $L^{2}$ as $\alpha \rightarrow \infty$.

Proof. By (1.2), we have

$$
\left|\nabla_{x} \Psi_{j}\left(y, t_{0}, \zeta\right)-\nabla_{x} \Psi_{j}\left(y, t_{0}, \xi\right)\right| \gtrsim|\zeta-\xi| .
$$

So, by scaling and the stationary phase method (see Lemma 11.1), we get

$$
\left|I_{j}^{\lambda, \alpha}(\zeta, \xi)\right| \lesssim(\alpha \lambda)^{n}(1+\alpha \lambda|\xi-\zeta|)^{-N_{0}}, \quad \forall N_{0} \geq 0 .
$$

First, we will show that $\widehat{f}_{\alpha} \rightarrow \widehat{f}$ a.e.

We write $\widehat{f}_{\alpha}$ as

$$
\widehat{f}_{\alpha}=\int_{|\xi-\zeta| \geq(\alpha \lambda)^{-1}} I_{j}^{\lambda, \alpha}(\zeta, \xi) \widehat{f}(\zeta) d \zeta+\int_{|\xi-\zeta|<(\alpha \lambda)^{-1}} I_{j}^{\lambda, \alpha}(\zeta, \xi) \widehat{f}(\zeta) d \zeta
$$

Using (6.1) and the Cauchy-Schwarz inequality, we obtain

$$
\begin{aligned}
\left|\int_{|\xi-\zeta| \geq(\alpha \lambda)^{-1}} I_{j}^{\lambda, \alpha}(\zeta, \xi) \widehat{f}(\zeta) d \zeta\right| & \lesssim(\alpha \lambda)^{n} \int_{|\xi-\zeta| \geq(\alpha \lambda)^{-1}}(1+\alpha|\xi-\zeta|)^{-N_{0}}|\widehat{f}(\zeta)| d \zeta \\
& \lesssim\|f\|_{2}\left(\frac{(\alpha \lambda)^{n}}{2 N_{0}-n}\right)^{1 / 2}
\end{aligned}
$$

If we take $N_{0}=(\alpha \lambda)^{n^{10}}$, then the last expression converges to zero as $\alpha \rightarrow \infty$. Next, let us write

$$
\begin{aligned}
& \int_{|\xi-\zeta|<(\alpha \lambda)^{-1}} I_{j}^{\lambda, \alpha}(\zeta, \xi) \widehat{f}(\zeta) d \zeta \\
& \quad=(\alpha \lambda)^{n} \int_{|\xi-\zeta|<(\alpha \lambda)^{-1}} \widehat{f}(\zeta) d \zeta+\int_{|\xi-\zeta|<(\alpha \lambda)^{-1}}\left(I_{j}^{\lambda, \alpha}(\zeta, \xi)-I_{j}^{\lambda, \alpha}(\xi, \xi)\right) \widehat{f}(\zeta) d \zeta .
\end{aligned}
$$

By the Lebesgue differentiation theorem, the first integral converges to $\widehat{f}$ a.e. as $\alpha \rightarrow \infty$. The kernel of the second integral is

$$
\left|I_{j}^{\lambda, \alpha}(\zeta, \xi)-I_{j}^{\lambda, \alpha}(\xi, \xi)\right| \lesssim \int\left|e^{i\left(\Psi_{j}^{\lambda}\left(y, t_{0}, \zeta\right)-\Psi_{j}^{\lambda}\left(y, t_{0}, \xi\right)\right)} a_{j}^{\lambda}(y / \alpha, \zeta)-a_{j}^{\lambda}(y / \alpha, \xi)\right| d y .
$$

Applying the mean-value theorem to the integrand, we have

$$
\left|I_{j}^{\lambda, \alpha}(\zeta, \xi)-I_{j}^{\lambda, \alpha}(\xi, \xi)\right| \lesssim(\alpha \lambda)^{n}|\zeta-\xi|
$$

Thus we have

$$
\begin{aligned}
\left|\int_{|\xi-\zeta|<(\alpha \lambda)^{-1}}\left(I_{j}^{\lambda, \alpha}(\zeta, \xi)-I_{j}^{\lambda, \alpha}(\xi, \xi)\right) \widehat{f}(\zeta) d \zeta\right| & \\
& \lesssim(\alpha \lambda)^{n} \int_{|\xi-\zeta|<(\alpha \lambda)^{-1}}|\zeta-\xi||\widehat{f}(\zeta)| d \zeta,
\end{aligned}
$$

which converges to zero a.e. as $\alpha \rightarrow \infty$ by the dominated convergence theorem. Thus we obtain $\widehat{f}_{\alpha} \rightarrow \widehat{f}$ a.e. 
It is not difficult to show that $f_{\alpha} \rightarrow f$ in $L^{2}$. From (6.1) we get

$$
\left|\widehat{f}_{\alpha}(\xi)\right| \lesssim M \widehat{f}(\xi),
$$

where $M$ denotes the Hardy-Littlewood maximal operator. Since both $M \widehat{f}$ and $\widehat{f}$ are in $L^{2}$, by the dominated convergence theorem we have

$$
\left\|\widehat{f}_{\alpha}-\widehat{f}\right\|_{2} \rightarrow 0 \quad \text { as } \quad \alpha \rightarrow \infty .
$$

Thus, by the Plancherel theorem we conclude that $f_{\alpha} \rightarrow f$ in $L^{2}$.

When considering $\mathcal{G}_{j}^{\lambda} f$, we may assume that the support of $\widehat{f}$ is contained in $\Xi_{j}$. We define a sequence $\mathcal{U}_{j}^{\lambda}=\mathcal{U}_{j}^{\lambda,\left(t_{0} ; \alpha\right)}$ by

$$
\mathcal{U}_{j}^{\lambda}=\mathcal{G}_{j}^{\lambda} f_{\left(t_{0} ; \alpha\right)} .
$$

By Fubini's theorem this is rewritten as

$$
\mathcal{U}_{j}^{\lambda}(x, t)=\int K_{j}^{\lambda, t_{0}}(x, t, y) U_{j}^{\lambda}\left(y, t_{0} ; \alpha\right) d y
$$

Here,

$$
K_{j}^{\lambda, t_{0}}(x, t, y)=\int e^{i\left(\Psi_{j}^{\lambda}(x, t, \xi)-\Psi_{j}^{\lambda}\left(y, t_{0}, \xi\right)\right)} a_{j}^{\lambda}(x, t, \xi) d \xi
$$

and

$$
U_{j}^{\lambda}(x, t ; \alpha)=\int e^{i \Psi_{j}^{\lambda}(x, t, \zeta)} a_{j}^{\lambda}\left(\alpha^{-1} x, t, \zeta\right) \widehat{f}(\zeta) d \zeta .
$$

If we define the operator $\mathcal{H}_{j}^{\lambda, t_{0}}$ by

$$
\mathcal{H}_{j}^{\lambda, t_{0}} f(x, t)=\int K_{j}^{\lambda, t_{0}}(x, t, y) f(y) d y,
$$

then we can write

$$
\mathcal{U}_{j}^{\lambda,\left(t_{0} ; \alpha\right)}=\mathcal{H}_{j}^{\lambda, t_{0}}\left[U_{j}^{\lambda}\left(t_{0} ; \alpha\right)\right]
$$

Note that we have

$$
\left|K_{j}^{\lambda, t_{0}}(z, y)\right| \lesssim\left(1+\operatorname{dist}\left(z, \Lambda_{j}^{\lambda,\left(y, t_{0}\right)}\right)\right)^{-N^{10}} .
$$

Indeed, let $\gamma^{(y, \xi)}$ be a curve given by the differential equation (1.7) with

$$
\gamma^{(y, \xi)}\left(t_{0} / \lambda\right)=y / \lambda
$$

This implies that

$$
\nabla_{\xi} \Psi_{j}\left(\gamma^{(y, \xi)}(t / \lambda), t / \lambda, \xi\right)=\nabla_{\xi} \Psi_{j}\left(y / \lambda, t_{0} / \lambda, \xi\right)
$$

for any $t \in \mathbb{R}$. From this and (1.2), we have

$$
\begin{aligned}
\left|\nabla_{\xi}\left(\Psi_{j}^{\lambda}(x, t, \xi)-\Psi_{j}^{\lambda}\left(y, t_{0}, \xi\right)\right)\right| & =\lambda\left|\nabla_{\xi} \Psi_{j}(x / \lambda, t / \lambda, \xi)-\nabla_{\xi} \Psi_{j}\left(\gamma^{(y, \xi)}(t / \lambda), t / \lambda, \xi\right)\right| \\
& \gtrsim\left|x-\lambda \gamma^{(y, \xi)}(t / \lambda)\right| .
\end{aligned}
$$

So, by the stationary phase method (see Lemma 11.1), we obtain (6.5). 
Lemma 6.2. Let $\lambda \geq R \geq C_{0}, \alpha \geq 1$ and $t, t_{0} \in \mathbb{R}$. Then we have that

$$
\begin{aligned}
\left\|U_{j}^{\lambda}\left(t_{0} ; \alpha\right)\right\|_{2} & \leq C, \\
\left\|\mathcal{H}_{j}^{\lambda, t_{0}} f(t)\right\|_{2} & \leq C\|f\|_{2}, \\
\left\|\mathcal{U}_{j}^{\lambda,\left(t_{0} ; \alpha\right)}\right\|_{L^{2}\left(Q_{R}\right)} & \leq C R^{1 / 2},
\end{aligned}
$$

where $j=1,2$ and the constants $C$ are independent of $t, t_{0}, \alpha$ and $\lambda$.

Proof. To show (6.6), we use the $T T^{*}$-method. Let

$$
T f(x)=\int e^{i \Psi_{j}^{\lambda}\left(x, t_{0}, \zeta\right)} a_{j}^{\lambda}\left(\alpha^{-1} x, t_{0}, \zeta\right) f(\zeta) d \zeta .
$$

Then

$$
T T^{*} f(x)=\int K(x, y) f(y) d y
$$

with

$$
K(x, y)=\int e^{i\left(\Psi_{j}^{\lambda}\left(x, t_{0}, \zeta\right)-\Psi_{j}^{\lambda}\left(y, t_{0}, \zeta\right)\right)} a_{j}^{\lambda}\left(\alpha^{-1} x, t_{0}, \zeta\right) \overline{a_{j}^{\lambda}\left(\alpha^{-1} y, t_{0}, \zeta\right)} d \zeta .
$$

It suffices to show that there is a constant $C$ such that

$$
\left\|T T^{*} f\right\|_{2} \leq C\|f\|_{2} .
$$

By Schur's lemma, this will follow from

$$
\int|K(x, y)| d x \leq C \quad \text { and } \quad \int|K(x, y)| d y \leq C .
$$

Using the stationary phase method (see Lemma 11.1), we obtain

$$
\begin{aligned}
|K(x, y)| & \lesssim(1+|x-y|)^{-N^{10}} \tilde{\chi}\left((\lambda \alpha)^{-1} x\right) \widetilde{\chi}\left((\lambda \alpha)^{-1} y\right) \\
& \lesssim(1+|x-y|)^{-N^{10}},
\end{aligned}
$$

where $\tilde{\chi}$ is a suitable bump function. From this we can get (6.9).

Consider (6.7). We use Schur's lemma again. From (6.3), it suffices to show that

$$
\int\left|K^{\lambda, t_{0}}(x, t, y)\right| d x \leq C \quad \text { and } \quad \int\left|K^{\lambda, t_{0}}(x, t, y)\right| d y \leq C .
$$

But these are just easy consequences of (6.5).

Inequality (6.8) follows easily from (6.6) and (6.7).

To exploit the spatial localization of $U_{j}^{\lambda}$, we will deal with $\mathcal{U}_{j}^{\lambda}$ instead of $U_{j}^{\lambda}$.

Definition 6.3. Let $D$ be a disk of radius $r>0$ with center $\left(x_{D}, t_{D}\right)$ and let $\mathcal{U}_{j}^{\lambda}=\mathcal{U}_{j}^{\lambda,\left(t_{D} ; \alpha\right)}$. We define a localization operator $L_{D} \mathcal{U}_{j}^{\lambda}$ as

$$
L_{D} \mathcal{U}_{j}^{\lambda}(z)=\mathcal{H}_{j}^{\lambda, t_{D}}\left[\left(\chi_{D} * \eta_{r^{1-1 / N}}\right) U_{j}^{\lambda}\left(t_{D} ; \alpha\right)\right],
$$

where $\chi_{D}$ is a characteristic function.

The following lemma shows that $L_{D} \mathcal{U}_{j}^{\lambda}$ is essentially supported in a neighborhood of the conic surface $\Lambda_{j}^{\lambda, z_{D}}$. 
Lemma 6.4. Let $\lambda \gtrsim r \geq C_{0}$ and $\alpha \geq 1$. Let $D$ be a disk of radius $r$ centered at $z_{D}=\left(x_{D}, t_{D}\right)$ and let $\mathcal{U}_{j}^{\lambda}=\mathcal{U}_{j}^{\lambda,\left(t_{D} ; \alpha\right)}$. Suppose that $q$ is a cube of side-length $r$ with center $z_{D}$ and that $\Lambda_{j}^{\lambda}$ is a conic surface with vertex $z_{D}$. Then we have

$$
\begin{gathered}
\left\|\left(1-L_{D}\right) \mathcal{U}_{j}^{\lambda}\right\|_{L^{\infty}(q)} \lesssim r^{C-N}, \\
\left\|L_{D} \mathcal{U}_{j}^{\lambda}\right\|_{L^{\infty}\left(\mathbb{R}^{n} \backslash \Lambda_{j}^{\lambda}\left(C r+\lambda^{1 / N}\right)\right)} \lesssim \lambda^{C-N} .
\end{gathered}
$$

Proof. To show (6.10), we note that by the Cauchy-Schwarz inequality and (6.6), it suffices to show that

$$
\int\left|K_{j}^{\lambda, t_{D}}(z, y)\left(1-\chi_{D} * \eta_{r^{1-1 / N}}\right)(y)\right|^{2} d y \lesssim r^{C-N},
$$

for any $z \in q$. Since $\Psi_{j} \in C^{\infty}$ is supported in a compact set, i.e. supp $a_{j}$, the slopes $d \gamma / d t$ of the conic surfaces $\Lambda_{j}^{\lambda}$ are bounded. Thus some geometric observations based on (6.5) imply that for any $z \in q$, we have

$$
\left(1-\operatorname{dist}\left(z, \Lambda_{j}^{\lambda,\left(y, t_{D}\right)}\right)\right)^{-N^{10}}\left(1-\chi_{D} * \eta_{r^{1-1 / N}}\right)(y) \lesssim r^{C-N} .
$$

From this, we obtain (6.12).

To prove (6.11), it suffices to show that

$$
\int\left|K_{j}^{\lambda, t_{D}}(z, y)\left(\chi_{D} * \eta_{r^{1-1 / N}}\right)(y)\right|^{2} d y \lesssim \lambda^{C-N},
$$

for any $z \in Q_{\lambda} \backslash \Lambda_{j}^{\lambda, z_{D}}\left(\mathrm{Cr}+\lambda^{1 / N}\right)$. By a similar observation we have that

$$
\left(1-\operatorname{dist}\left(z, \Lambda_{j}^{\lambda,\left(y, t_{D}\right)}\right)\right)^{-N^{10}}\left(\chi_{D} * \eta_{r^{1-1 / N}}\right)(y) \lesssim \lambda^{C-N},
$$

for any $z \in Q_{\lambda} \backslash \Lambda_{j}^{\lambda, z_{D}}\left(\mathrm{Cr}+\lambda^{1 / N}\right)$. So we obtain (6.13).

\section{ENERGy CONCENTRATION}

We begin with the definition of an energy-concentrated disc.

Definition 7.1. Let $\lambda>2^{C_{0}}$ and $-\lambda \leq t_{e} \leq \lambda$. For two given numbers $0<\delta \ll 1$ and $\alpha \geq 1$, we define an energy-concentrated disc $D_{e}$ to be a largest disc of radius $r_{e} \geq 2^{\bar{C}_{0}(1 / 2+4 / N)}$ with center $z_{e}=\left(x_{e}, t_{e}\right)$ such that

$$
1-2 \delta<\frac{\left\|U_{1}^{\lambda}(\cdot ; \alpha)\right\|_{L^{2}\left(D_{e}\right)}\left\|U_{2}^{\lambda}(\cdot ; \alpha)\right\|_{L^{2}\left(D_{e}\right)}}{\left\|U_{1}^{\lambda}\left(t_{e} ; \alpha\right)\right\|_{L_{x}^{2}}\left\|U_{2}^{\lambda}\left(t_{e} ; \alpha\right)\right\|_{L_{x}^{2}}}<1-\delta .
$$

To consider the energy concentration, we modify Hypothesis 5.1 as the following:

Hypothesis 7.2. Let $\lambda \geq R>2^{C_{0}},-\lambda<t_{e}<\lambda$ and $\alpha \geq 1$. We define $A^{\lambda}(R)$ to be the least constant such that the inequality

$$
\left\|\mathcal{U}_{1}^{\lambda,\left(t_{e} ; \alpha\right)} \mathcal{U}_{2}^{\lambda,\left(t_{e} ; \alpha\right)}\right\|_{L^{p}\left(Q_{R}\right)} \leq A^{\lambda}(R) E\left(\mathcal{U}_{1}^{\lambda,\left(t_{e} ; \alpha\right)}\right)^{1 / 2} E\left(\mathcal{U}_{2}^{\lambda,\left(t_{e} ; \alpha\right)}\right)^{1 / 2}
$$

holds for any cube $Q_{R} \subset \mathbb{R}^{n+1}$ of side-length $R$.

Here, the energy $E\left(\mathcal{H}_{j}^{\lambda, t_{e}} f\right)$ is defined by

$$
E\left(\mathcal{H}_{j}^{\lambda, t_{e}} f\right):=\|f\|_{2}^{2} .
$$


So, $E\left(\mathcal{U}_{1}^{\lambda,\left(t_{e} ; \alpha\right)}\right)=\left\|U_{1}^{\lambda}\left(t_{e} ; \alpha\right)\right\|_{L_{x}^{2}}^{2}$. Note that by the perturbation of phases we have $A^{\lambda}(R) \lesssim A(R)$. Let $\mathcal{U}_{j}^{\lambda}$ stand for $\mathcal{U}_{j}^{\lambda,\left(t_{e} ; \alpha\right)}$ from now on, unless explicitly stated otherwise.

Lemma 7.3. Suppose that $D$ is a disk of radius $r \geq C_{0}^{1 / 2} r_{e}$ and centered at $z_{e}$. Then we have

$$
\left\|\left(1-L_{D}\right) \mathcal{U}_{1}^{\lambda}\left(1-L_{D}\right) \mathcal{U}_{2}^{\lambda}\right\|_{p} \lesssim\left(\delta+C_{0}^{-C}\right) A^{\lambda}(\lambda) .
$$

Proof. By Hypothesis 7.2 , it suffices to show that

$$
E\left(\left(1-L_{D}\right) \mathcal{U}_{1}\right)^{1 / 2} E\left(\left(1-L_{D}\right) \mathcal{U}_{2}\right)^{1 / 2} \lesssim \delta+C_{0}^{-C} .
$$

We have

$$
E\left(\left(1-L_{D}\right) \mathcal{U}_{j}\right)^{1 / 2}=\left\|\left(1-\chi_{D} * \eta_{r_{e}^{1-1 / N}}\right) U_{j}\left(t_{e} ; \alpha\right)\right\|_{2} .
$$

A pointwise estimate gives

$$
\left\|\left(1-\chi_{D} * \eta_{r_{e}^{1-1 / N}}\right) U_{j}\left(t_{e} ; \alpha\right)\right\|_{2}^{2} \lesssim\left\|U_{j}\left(t_{e} ; \alpha\right)\right\|_{L^{2}\left(\mathbb{R}^{n} \backslash D\right)}^{2}+C r_{e}^{-N}\left\|U_{j}\left(t_{e} ; \alpha\right)\right\|_{2}^{2} .
$$

From (7.1), we have

$$
\left\|U_{j}^{\lambda}(\cdot ; \alpha)\right\|_{L^{2}\left(D_{e}\right)}^{2} \geq(1-4 \delta)\left\|U_{j}^{\lambda}\left(t_{e} ; \alpha\right)\right\|_{2}^{2}, \quad j=1,2,
$$

because $0 \leq\left\|U_{j}^{\lambda}(\cdot ; \alpha)\right\|_{L^{2}\left(D_{e}\right)} /\left\|U_{j}^{\lambda}\left(t_{e} ; \alpha\right)\right\|_{L_{x}^{2}} \leq 1$. So, we have

$$
\left\|U_{j}\left(t_{e} ; \alpha\right)\right\|_{L^{2}\left(\mathbb{R}^{n} \backslash D\right)}^{2} \lesssim \delta\left\|U_{j}\left(t_{e} ; \alpha\right)\right\|_{2}^{2} .
$$

Since $r_{e}>2^{C_{0} / 2}$, we thus obtain that

$$
E\left(\left(1-L_{D}\right) \mathcal{U}_{j}\right) \lesssim\left(\delta+C_{0}^{-C}\right)\left\|U_{j}\left(t_{e} ; \alpha\right)\right\|_{2}^{2} \lesssim \delta+C_{0}^{-C},
$$

from which we get (7.3).

First, we will localize $\mathcal{U}_{1}^{\lambda} \mathcal{U}_{2}^{\lambda}$ to some cube whose center is $z_{e}$. For this, we need the following lemma, whose proof is similar to that of (3.6) in Proposition 3.2

Lemma 7.4. Let $2^{C_{0}} \leq l<\lambda / 2$ and $2^{C_{0}(1 / 2+4 / N)}<r<l^{1 / 2+4 / N}$. Let $D$ be the disc of radius $C_{0}^{1 / 2} r$ with center $z_{D}=\left(x_{D}, t_{D}\right)$ and let $\mathcal{U}_{j}^{\lambda}=\mathcal{U}_{j}^{\lambda,\left(t_{D} ; \alpha\right)}$. Suppose that $Q_{l}$ is a cube of side-length $l$, whose center is that of $D$. Then we have

$$
\left\|\left(L_{D} \mathcal{U}_{1}^{\lambda}\right) \mathcal{U}_{2}^{\lambda}\right\|_{L^{p}\left(Q_{2 l} \backslash Q_{l}\right)} \lesssim l^{-1 / C} .
$$

Proof. By interpolation it suffices to show the following two estimates:

$$
\begin{aligned}
\left\|\left(L_{D} \mathcal{U}_{1}\right) \mathcal{U}_{2}\right\|_{L^{1}\left(Q_{2 l} \backslash Q_{l}\right)} \leq C l^{\varepsilon} l^{\frac{3}{4}} \\
\left\|\left(L_{D} \mathcal{U}_{1}\right) \mathcal{U}_{2}\right\|_{L^{2}\left(Q_{2 l} \backslash Q_{l}\right)} \leq C l^{\varepsilon} l^{\frac{-n+1}{4}},
\end{aligned}
$$

where the constants $C$ are independent of $\varepsilon$. In this proof, all constants $C$ do not depend on $\varepsilon$, and neither do the implied constants associated with the symbol $\lesssim$.

We first consider (7.4). From Lemma 6.4 and the Cauchy-Schwarz inequality, we see that

$$
\begin{aligned}
\left\|\left(L_{D} \mathcal{U}_{1}\right) \mathcal{U}_{2}\right\|_{L^{1}\left(Q_{2 l} \backslash \Lambda_{1}^{\lambda}\left(C C_{0}^{1 / 2} l^{1 / 2+\varepsilon}\right)\right)} & \lesssim l^{-N}\left\|\mathcal{U}_{2}\right\|_{L^{1}\left(Q_{2 l} \backslash \Lambda_{1}^{\lambda}\left(C C_{0}^{1 / 2} l^{1 / 2+\varepsilon}\right)\right)} \\
& \lesssim l^{-N+C}\left\|\mathcal{U}_{2}\right\|_{L^{2}\left(Q_{2 l}\right)} .
\end{aligned}
$$

By (6.8), we thus have

$$
\left\|\left(L_{D} \mathcal{U}_{1}\right) \mathcal{U}_{2}\right\|_{L^{1}\left(Q_{2 l} \backslash \Lambda_{1}^{\lambda}\left(C C_{0}^{1 / 2} l^{1 / 2+\varepsilon}\right)\right)} \lesssim l^{-N+C} .
$$


By the Cauchy-Schwarz inequality, we have

$$
\left\|\left(L_{D} \mathcal{U}_{1}\right) \mathcal{U}_{2}\right\|_{L^{1}\left(\left(Q_{2 l} \backslash Q_{1}\right) \cap \Lambda_{1}^{\lambda}\left(C C_{0}^{1 / 2} l^{1 / 2+\varepsilon}\right)\right)} \lesssim\left\|\left(L_{D} \mathcal{U}_{1}\right)\right\|_{L^{2}\left(Q_{2 l}\right)}\left\|\mathcal{U}_{2}\right\|_{L^{2}\left(\Lambda_{1}^{\lambda}\left(C C_{0}^{1 / 2} l^{1 / 2+\varepsilon}\right)\right)},
$$

and by (6.6), (6.7) and Proposition 2.2 we get

$$
\left\|\left(L_{D} \mathcal{U}_{1}\right) \mathcal{U}_{2}\right\|_{L^{1}\left(\left(Q_{2 l} \backslash Q_{1}\right) \cap \Lambda_{1}^{\lambda}\left(C C_{0}^{1 / 2} l^{1 / 2+\varepsilon}\right)\right)} \lesssim l^{\varepsilon} l^{\frac{3}{4}} .
$$

From this and (77.6), we obtain (7.4).

To prove (7.5), we decompose $L_{D} \mathcal{U}_{1}$ into wavepackets. Here, we do not need to consider the Schwartz tails so carefully; hence we may use the usual wavepacket decomposition. Let

$$
u_{(w, v)}(z)=\int K_{v}^{t_{D}}(z, y)\left(\chi_{D} * \eta_{r^{1-1 / N}}\right)(y) \eta\left(l^{-1 / 2}(y-w)\right) U_{1}\left(y, t_{D} ; \alpha\right) d y,
$$

where

$$
K_{v}^{t_{D}}(z, y)=\int e^{i\left(\Psi_{1}^{\lambda}(x, t, \xi)-\Psi_{1}^{\lambda}\left(y, t_{D}, \xi\right)\right)} a_{j}^{\lambda}(x, t, \xi) \rho\left(l^{1 / 2}(\xi-v)\right) d \xi .
$$

Then we have

$$
L_{D} \mathcal{U}_{1}=\sum_{(w, v) \in G \times V_{1}} u_{(w, v)},
$$

where $G=l^{1 / 2} \mathbb{Z}^{n}$ and $V_{1}=\operatorname{supp}_{\xi}\left(S_{1}\right) a_{1} \cap l^{-1 / 2} \mathbb{Z}^{n}$.

Note that by the stationary phase method (see Lemma 11.1), we have

$$
\left|K_{v}^{t_{D}}(z, y)\right| \lesssim l^{-n / 2}\left(1+l^{-1 / 2}\left(x-\lambda \gamma_{1}^{(y, v)}(t / \lambda)\right)\right)^{-N^{10}},
$$

where $\gamma_{1}^{(y, v)}$ is the curve defined by the differential equation (1.7) with

$$
\lambda \gamma_{1}^{(y, v)}\left(t_{D} / \lambda\right)=y
$$

First, when $\left|w-x_{D}\right| \gg l$, the term $\left(\chi_{D} * \eta_{r^{1-1 / N}}\right)(y) \eta\left(l^{-1 / 2}(y-w)\right)$ is almost zero, and when $l^{1 / 2+\varepsilon} \lesssim\left|w-x_{D}\right| \lesssim l$, we have that $\left(\chi_{D} * \eta_{r^{1-1 / N}}\right)(y) \eta\left(l^{-1 / 2}(y-w)\right) \lesssim$ $l^{-N}$. So, by the Cauchy-Schwarz inequality, we have

$$
\left|u_{(w, v)}(z)\right| \lesssim l^{C-N} .
$$

Since the number of such $u_{(w, v)}$ is only $\mathcal{O}\left(l^{C}\right)$, we can disregard these wavepackets in (7.7). Now it suffices to show that

$$
\left\|\sum_{\substack{(w, v) \in G \times V_{1}: \\\left|w-x_{D}\right| \lesssim l^{1 / 2+\varepsilon}}} u_{(w, v)} \mathcal{U}_{2}\right\|_{L^{2}\left(Q_{2 l} \backslash Q_{l}\right)} \lesssim l^{\varepsilon} l^{-\frac{n-1}{4}} .
$$

Using (7.8), we have

$$
\begin{aligned}
& \left|u_{(w, v)}\right| \\
& \lesssim l^{-n / 2}\left(1+l^{-1 / 2}\left(x-\lambda \gamma_{1}^{(w, v)}(t / \lambda)\right)\right)^{-N^{10}} \int\left|U_{1}\left(y, t_{D} ; \alpha\right)\right| \eta\left(l^{-1 / 2}(y-w)\right) d y .
\end{aligned}
$$

From the Cauchy-Schwarz inequality and (6.6), it follows that

$$
\int\left|U_{1}\left(y, t_{D} ; \alpha\right)\right| \eta\left(l^{-1 / 2}(y-w)\right) d y \lesssim l^{n / 4}\left\|U_{1}^{\lambda}\left(\cdot, t_{D} ; \alpha\right)\right\|_{2} \lesssim l^{n / 4} .
$$


So it follows that

$$
\left|u_{(w, v)}\right| \lesssim l^{-n / 4}\left(1+l^{-1 / 2}\left(x-\lambda \gamma_{1}^{(w, v)}(t / \lambda)\right)\right)^{-N^{10}} .
$$

Since the number of $w$ such that $\left|w-x_{D}\right| \lesssim l^{1 / 2+\varepsilon}$ is about $l^{\varepsilon}$, it now suffices to show that

$$
\left\|\sum_{v \in V_{1}}\left(1+l^{-1 / 2}\left(x-\lambda \gamma_{1}^{\left(x_{D}, v\right)}(t / \lambda)\right)\right)^{-N^{10}} \mathcal{U}_{2}\right\|_{L^{2}\left(Q_{2 l} \backslash Q_{l}\right)} \lesssim l^{\varepsilon} l^{1 / 4} .
$$

Since $\operatorname{dist}\left(x_{D}, Q_{2 l} \backslash Q_{l}\right) \gtrsim l$, we have

$$
\sum_{v \in V_{1}}\left(1+l^{-1 / 2}\left(x-\lambda \gamma_{1}^{\left(x_{D}, v\right)}(t / \lambda)\right)\right)^{-N^{10}} \lesssim\left(1+l^{-1 / 2} \operatorname{dist}\left(z, \Lambda_{1}^{\lambda, z_{D}}\right)\right)^{-N^{10}} .
$$

So the left side of (7.9) has the bound

$$
\left\|\sum_{v \in V_{1}}\left(1+l^{-1 / 2}\left(x-\lambda \gamma_{1}^{\left(x_{D}, v\right)}(t / \lambda)\right)\right)^{-N^{10}} \mathcal{U}_{2}\right\|_{L^{2}\left(Q_{2 l} \backslash Q_{l}\right)} \lesssim\left\|\mathcal{U}_{2}\right\|_{L^{2}\left(\Lambda_{1}^{\lambda}\left(l^{1 / 2+\varepsilon}\right)\right)} .
$$

By Proposition 2.2 and (6.8), we thus obtain (7.9).

Proposition 7.5. Let $\lambda \geq 2^{C_{0}}$ and $R=r_{e}^{1 /(1 / 2+4 / N)}$. Suppose that $Q_{R}^{e}$ is the cube centered at $z_{e}$ with side-length $R$. Then we have

$$
\left\|\mathcal{U}_{1}^{\lambda} \mathcal{U}_{2}^{\lambda}\right\|_{L^{p}\left(\mathbb{R}^{n+1} \backslash Q_{R}^{e}\right)} \lesssim\left(\delta+C_{0}^{-C}\right) A^{\lambda}(\lambda)+R^{-1 / C} .
$$

Proof. Let $\widetilde{D}$ be a disk of radius $C_{0}^{1 / 2} r_{e}$, centered at $z_{e}$. By the triangle inequality, the desired estimate will follow from the following three estimates:

$$
\begin{aligned}
\left\|\left(1-L_{\widetilde{D}}\right) \mathcal{U}_{1}^{\lambda}\left(1-L_{\widetilde{D}}\right) \mathcal{U}_{2}^{\lambda}\right\|_{p} & \lesssim\left(\delta+C_{0}^{-C}\right) A^{\lambda}(\lambda), \\
\left\|\left(L_{\widetilde{D}} \mathcal{U}_{1}^{\lambda}\right) \mathcal{U}_{2}^{\lambda}\right\|_{L^{p}\left(\mathbb{R}^{n+1} \backslash Q_{R}^{e}\right)} & \lesssim R^{-1 / C}, \\
\left\|\left(1-L_{\widetilde{D}}\right) \mathcal{U}_{1} L_{\widetilde{D}} \mathcal{U}_{2}\right\|_{L^{p}\left(\mathbb{R}^{n+1} \backslash Q_{R}^{e}\right)} & \lesssim R^{-1 / C} .
\end{aligned}
$$

From Lemma 7.3, we have (7.10). To show (7.11), we first decompose $Q_{\alpha \lambda} \backslash Q_{R}^{e}$ dyadically. By applying Lemma 7.4 to each piece, we obtain (7.11). In a similar way, we obtain (7.12). By replacing $L_{D} \mathcal{U}_{1}^{\lambda}$ and $\mathcal{U}_{2}^{\lambda}$ with $L_{D} \mathcal{U}_{2}^{\lambda}$ and $\left(1-L_{D}\right) \mathcal{U}_{1}^{\lambda}$ in Lemma 7.4 respectively, we may get the same estimate.

Next, we will show that most values of $\mathcal{U}_{1}^{\lambda} \mathcal{U}_{2}^{\lambda}$ are contained in a neighborhood of some conic surface whose vertex is in the energy-concentrated portion.

Proposition 7.6. Let $\lambda \geq 2^{C_{0}}$. Then we have

$$
\left\|\mathcal{U}_{1}^{\lambda} \mathcal{U}_{2}^{\lambda}\right\|_{L^{p}\left(\mathbb{R}^{n+1} \backslash \Lambda^{\lambda, z_{e}}\left(C_{0} r_{e}+C_{0}\right)\right)} \lesssim\left(\delta+C_{0}^{-C}\right) A^{\lambda}(\lambda) .
$$

Proof. Let $\widetilde{D}$ be a disk of radius $C_{0}^{1 / 2} r_{e}$ and center $z_{e}$. By the triangle inequality, it suffices to show the following three estimates:

$$
\begin{gathered}
\left\|\left(1-L_{\widetilde{D}}\right) \mathcal{U}_{1}^{\lambda}\left(1-L_{\widetilde{D}}\right) \mathcal{U}_{2}^{\lambda}\right\|_{p} \lesssim\left(\delta+C_{0}^{-C}\right) A^{\lambda}(\lambda), \\
\left\|\left(L_{\widetilde{D}} \mathcal{U}_{1}^{\lambda}\right) \mathcal{U}_{2}^{\lambda}\right\|_{L^{p}\left(\mathbb{R}^{n+1} \backslash \Lambda^{\lambda, z_{e}}\left(C_{0} r_{e}+C_{0}\right)\right)} \lesssim C_{0}^{-C}, \\
\left\|\left(1-L_{\widetilde{D}}\right) \mathcal{U}_{1}^{\lambda} L_{\widetilde{D}} \mathcal{U}_{2}^{\lambda}\right\|_{L^{p}\left(\mathbb{R}^{n+1} \backslash \Lambda^{\lambda, z_{e}}\left(C_{0} r_{e}+C_{0}\right)\right)} \lesssim C_{0}^{-C} .
\end{gathered}
$$


By Lemma 7.3, we have (7.13). The proofs of estimates (7.14) and (7.15) are similar. So we only need to show (7.14). By (6.11) in Lemma 6.4 we have

$$
\left\|\left(L_{\widetilde{D}} \mathcal{U}_{1}^{\lambda}\right) \mathcal{U}_{2}^{\lambda}\right\|_{L^{p}\left(\mathbb{R}^{n+1} \backslash \Lambda^{\lambda, z_{e}}\left(C_{0} r_{e}+C_{0}\right)\right)} \lesssim \lambda^{-N}\left\|\mathcal{U}_{2}^{\lambda}\right\|_{p}
$$

and by Hölder's inequality we get

$$
\left\|\mathcal{U}_{2}^{\lambda}\right\|_{p} \lesssim \lambda^{C}\left\|\mathcal{U}_{2}^{\lambda}\right\|_{2}
$$

Hence it follows that

$$
\left\|\left(L_{\widetilde{D}} \mathcal{U}_{1}^{\lambda}\right) \mathcal{U}_{2}^{\lambda}\right\|_{L^{p}\left(\mathbb{R}^{n+1} \backslash \Lambda^{\lambda, z_{e}}\left(C_{0} r_{e}+C_{0}\right)\right)} \lesssim \lambda^{-N+C} .
$$

Since $\lambda>C_{0}$ and $N$ are large, (7.14) follows.

Corollary 7.7. We assume that $\left\|\mathcal{U}_{1}^{\lambda} \mathcal{U}_{2}^{\lambda}\right\|_{p} \sim A^{\lambda}(\lambda)$. Let $R=r_{e}^{1 /(1 / 2+4 / N)}$ and let $Q_{R}^{e}$ be the cube centered at $z_{e}$ with side-length $R$. Then we have

$$
\left\|\mathcal{U}_{1}^{\lambda} \mathcal{U}_{2}^{\lambda}\right\|_{p} \leq\left(1-C\left(\delta+C_{0}^{-C}\right)^{p}\right)^{-1 / p}\left\|\mathcal{U}_{1}^{\lambda} \mathcal{U}_{2}^{\lambda}\right\|_{L^{p}\left(\Omega^{e}\right)}
$$

where $\Omega^{e}=Q_{R}^{e} \cap \Lambda^{\lambda, z_{e}}\left(C_{0} r_{e}+C_{0}\right)$.

Proof. In Proposition 7.5, the $R^{-1 / C}$ term can be absorbed into the main term because $R \geq 2^{C_{0}}$. Combining Proposition 7.5 and Proposition 7.6 we have

$$
\left\|\mathcal{U}_{1}^{\lambda} \mathcal{U}_{2}^{\lambda}\right\|_{L^{p}\left(\mathbb{R}^{n+1} \backslash \Omega^{e}\right)} \lesssim\left(\delta+C_{0}^{-C}\right) A^{\lambda}(\lambda)
$$

By our assumption, we have

$$
\left\|\mathcal{U}_{1}^{\lambda} \mathcal{U}_{2}^{\lambda}\right\|_{L^{p}\left(\mathbb{R}^{n+1} \backslash \Omega^{e}\right)} \lesssim\left(\delta+C_{0}^{-C}\right)\left\|\mathcal{U}_{1}^{\lambda} \mathcal{U}_{2}^{\lambda}\right\|_{p} .
$$

By a rearrangement after taking the $p$-th power of the above inequality, we get

$$
\left\|\mathcal{U}_{1}^{\lambda} \mathcal{U}_{2}^{\lambda}\right\|_{p} \leq\left(1-C\left(\delta+C_{0}^{-C}\right)^{p}\right)^{-1 / p}\left\|\mathcal{U}_{1}^{\lambda} \mathcal{U}_{2}^{\lambda}\right\|_{L^{p}\left(\Omega^{e}\right)}
$$

\section{Modification of SECtion 3}

In this section, we modify the wave packets to obtain a specific constant $1+C c$ in Lemma 9.2 below. First, we define operators $\mathfrak{F}_{j}$ and $\mathfrak{F}_{j}^{*}$ by

$$
\begin{aligned}
& \mathfrak{F}_{j}^{*} f(x)=\mathfrak{F}_{j}^{\lambda, t_{e}} f(x)=\int e^{i \psi_{j}^{\lambda}\left(x, t_{e}, \xi\right)} f(\xi) a_{\Xi_{j}}(\xi) d \xi, \\
& \mathfrak{F}_{j} f(\xi)=\mathfrak{F}_{j}^{\lambda, t_{e}} f(\xi)=\int e^{-i \psi_{j}^{\lambda}\left(x, t_{e}, \xi\right)} f(x) d x .
\end{aligned}
$$

Using these, we can rewrite

$$
\begin{gathered}
U_{j}^{\lambda}\left(x, t_{e} ; \alpha\right)=a_{\times}^{\lambda}\left(\alpha^{-1} x\right) \mathfrak{F}_{j}^{*} \widehat{f}(x), \\
\widehat{f}_{\alpha}=\mathfrak{F}_{j}\left(a_{\times}^{\alpha \lambda} \mathfrak{F}_{j}^{*} \widehat{f}\right), \\
\mathcal{U}_{j}^{\lambda}=\mathcal{G}_{j}^{\lambda} f_{\alpha}=\mathcal{G}_{j}^{\lambda} \mathcal{F}^{-1} \mathfrak{F}_{j}\left(a_{\times}^{\alpha \lambda} \mathfrak{F}_{j}^{*} \widehat{f}\right), \\
\mathcal{H}_{j}^{\lambda, t_{e}}=\mathcal{G}_{j}^{\lambda} \mathcal{F}^{-1} \mathfrak{F}_{j},
\end{gathered}
$$

where $\mathcal{F}^{-1}$ denotes the inverse Fourier transform.

When defining new wave packets, we will use the operators $\mathfrak{F}_{j}, \mathfrak{F}_{j}^{*}$ instead of the Fourier transforms $\mathcal{F}, \mathcal{F}^{-1}$, respectively. Employing the notation in section 3 , we define $u_{T_{j}}(x)$ for each $T_{j}=(y, v) \in G^{R} \times V_{j}^{R}$ by

$$
u_{T_{j}}(x):=a_{\times}^{\lambda}\left(\alpha^{-1} x\right) \eta_{y}(x) \mathfrak{F}_{j}^{*}\left(\rho_{v} \widehat{f}\right)(x),
$$


and define a wave packet $\mathcal{U}_{T_{j}}^{\lambda}$ by

$$
\mathcal{U}_{T_{j}}^{\lambda}:=\mathcal{H}_{j}^{\lambda, t_{e}} u_{T_{j}} .
$$

Then we have a decomposition

$$
\mathcal{U}_{j}^{\lambda}=\sum_{T_{j} \in \mathcal{T}_{j}^{R}} \mathcal{U}_{T_{j}}^{\lambda},
$$

where $T_{j}=(y, v)$ and $\mathcal{T}_{j}^{R}=G^{R} \times V_{j}^{R}$.

For each cube $b \in \mathcal{Q}_{C_{0}}\left(\widetilde{Q}_{R}\right)$, we define $U_{j}^{(b)}\left(x, t_{e} ; \alpha\right)=U_{j}^{(b), V}\left(x, t_{e} ; \alpha\right)$ by

$$
U_{j}^{(b)}\left(x, t_{e} ; \alpha\right)=\sum_{T_{j}} m_{T_{j}}^{(b)} u_{T_{j}}(x)
$$

and define $\mathcal{U}_{j}^{(b)}$ by

$$
\mathcal{U}_{j}^{(b)}=\mathcal{H}_{j}^{\lambda, t_{e}}\left[U_{j}^{(b)}\left(t_{e} ; \alpha\right)\right]
$$

where the constant $m_{T_{j}}^{(b)}=\left\|\tilde{\chi}_{T_{j}} V\right\|_{L^{2}(b)}^{2} /\left\|\tilde{\chi}_{T_{j}} V\right\|_{L^{2}\left(\widetilde{Q}_{R}\right)}^{2}$ and $\tilde{\chi}_{T_{j}}$ is a bump function which is essentially supported on the $O\left(R^{1 / 2}\right)$-neighborhood of a curve $\left(\lambda \gamma_{j}^{v}(t / \lambda), t\right)$ with $\lambda \gamma_{j}^{v}\left(t_{e} / \lambda\right)=y$. Thus we have a decomposition

$$
\mathcal{U}_{j}^{\lambda}=\sum_{b \in \mathcal{Q}_{C_{0}}\left(\widetilde{Q}_{R}\right)} \mathcal{U}_{j}^{(b)} .
$$

Our new wave packets also satisfy the properties in Lemma 3.1 .

Lemma 8.1. Let

$$
h_{(y, v)}=R^{n / 4} M\left(\mathfrak{F}_{j}^{*}\left(\rho_{v} \widehat{f}\right)\right)(y) \quad \text { and } \quad \phi_{(y, v)}=h_{(y, v)}^{-1} \mathcal{U}_{(y, v)}^{\lambda} .
$$

Suppose that $0<c \leq 2^{-C_{0}}$ and $\alpha \geq 1$.

(1) For each $(y, v) \in G^{R} \times V_{j}^{R}$, we have

$$
\left|\phi_{(y, v)}(x, t)\right| \lesssim c^{-C} R^{-n / 4}\left(1+\frac{\left|x-\lambda \gamma_{j}^{v}(t / \lambda)\right|}{R^{1 / 2}}\right)^{-N^{10}}
$$

where $\gamma_{j}^{v}(t)=\gamma_{j}^{(y, v)}(t)$ satisfies $\gamma_{j}^{(y, v)}\left(t_{e}\right)=y / \lambda$.

(2) If the Fourier support of $f$ is contained in $\operatorname{supp}_{\xi} a_{j}$, then we have

$$
\left(\sum_{(y, v) \in G^{R} \times V_{j}^{R}}\left|h_{(y, v)}\right|^{2}\right)^{1 / 2} \lesssim\|f\|_{2} .
$$

Proof. The arguments are almost the same as those of Lemma 3.1 .

We will show (11). From (8.2), (8.3) and (8.4), we have

$$
\mathcal{U}_{T_{j}}^{\lambda}(z)=\mathcal{G}_{j}^{\lambda} \mathcal{F}^{-1} \mathfrak{F}_{j} u_{T_{j}} .
$$

Consider $\mathfrak{F}_{j} u_{T_{j}}=\mathfrak{F}_{j}\left[a_{\times}^{\alpha \lambda} \eta_{y} \mathfrak{F}_{j}^{*}\left(\rho_{v} \widehat{f}\right)\right]$. We can write

$$
\mathfrak{F}_{j}\left[\eta_{y} \mathfrak{F}_{j}^{*}\left(\rho_{v} \widehat{f}\right)\right](\xi)=\int K(\xi, \zeta) \rho_{v}(\zeta) \widehat{f}(\zeta) d \zeta,
$$

where

$$
K(\xi, \zeta)=\int e^{i \psi_{j}^{\lambda}\left(x, t_{e}, \zeta\right)-i \psi_{j}^{\lambda}\left(x, t_{e}, \xi\right)} \eta_{y}(x) a_{\times}^{\lambda}\left(\alpha^{-1} x\right) d x
$$


Since

we obtain that

$$
\left|\nabla_{x} \psi_{j}^{\lambda}\left(x, t_{e}, \zeta\right)-\nabla_{x} \psi_{j}^{\lambda}\left(x, t_{e}, \xi\right)\right| \sim|\zeta-\xi|
$$

$$
|K(\xi, \zeta)| \lesssim\left(c^{-2} R^{1 / 2}\right)^{n}\left(1+c^{-2} R^{1 / 2}|\xi-\zeta|\right)^{-N^{10}}
$$

by the stationary phase method (see Lemma 11.1). From these estimates we can observe that the support of $\mathfrak{F}_{j} u_{T_{j}}$ is contained in the $O\left(\left(1+c^{2}\right)^{-1} R^{1 / 2}\right)$-neighborhood of $v$. Thus, we can insert a smooth bump function $\widetilde{\rho}_{v}(\xi)=\rho\left(\left(1+c^{2}\right)^{-1} R^{1 / 2}(\xi-v)\right)$ into $\mathcal{U}_{(y, v)}^{\lambda}$, i.e.,

$$
\mathcal{U}_{(y, v)}^{\lambda}=\mathcal{G}_{j}^{\lambda} \mathcal{F}^{-1}\left[\widetilde{\rho}_{v} \mathfrak{F}_{j} u_{T_{j}}\right] .
$$

Then, by rearranging the integrals we have

$$
\mathcal{U}_{(y, v)}^{\lambda}(z)=\int K_{v}\left(z, x^{\prime}\right) u_{T_{j}}\left(x^{\prime}\right) d x^{\prime},
$$

where

$$
K_{v}\left((x, t), x^{\prime}\right)=\int e^{i\left(\Psi_{j}^{\lambda}(x, t, \xi)-\Psi_{j}^{\lambda}\left(x^{\prime}, t_{e}, \xi\right)\right)} a_{j}^{\lambda}(x, t, \xi) \widetilde{\rho}_{v}(\xi) d \xi .
$$

Applying the stationary phase method (see Lemma 11.1), we get

$$
\begin{aligned}
& \left|K_{v}\left((x, t), x^{\prime}\right)\right| \\
& \quad \lesssim\left(\left(1+c^{2}\right) R^{-1 / 2}\right)^{n}\left(1+\frac{\lambda\left|\nabla_{\xi} \Psi_{j}(x / \lambda, t / \lambda, v)-\nabla_{\xi} \Psi_{j}\left(x^{\prime} / \lambda, t_{e} / \lambda, v\right)\right|}{\left(1+c^{2}\right)^{-1} R^{1 / 2}}\right)^{-N^{10}} .
\end{aligned}
$$

From the initial condition $\gamma_{j}^{\left(x^{\prime}, v\right)}\left(t_{e}\right)=x^{\prime} / \lambda$ and (1.8), we have

$$
\begin{aligned}
\mid \nabla_{\xi} \Psi_{j}(x / \lambda, t / \lambda, v)-\nabla_{\xi} \Psi_{j} & \left(x^{\prime} / \lambda, t_{e} / \lambda,, v\right) \mid \\
& =\left|\nabla_{\xi} \Psi_{j}(x / \lambda, t / \lambda, v)-\nabla_{\xi} \Psi_{j}\left(\gamma_{j}^{\left(x^{\prime}, v\right)}(t / \lambda), t / \lambda, v\right)\right| \\
& \sim\left|x / \lambda-\gamma_{j}^{\left(x^{\prime}, v\right)}(t / \lambda)\right| .
\end{aligned}
$$

Thus, we obtain

$$
\left|K_{v}\left((x, t), x^{\prime}\right)\right| \lesssim\left(\left(1+c^{2}\right) R^{-1 / 2}\right)^{n}\left(1+\frac{\left|x-\lambda \gamma_{j}^{\left(x^{\prime}, v\right)}(t / \lambda)\right|}{\left(1+c^{2}\right)^{-1} R^{1 / 2}}\right)^{-N^{10}},
$$

from which we have

$$
\left|\mathcal{U}_{(y, v)}^{\lambda}(x, t)\right| \lesssim c^{-C}\left(1+\frac{\left|x-\lambda \gamma_{j}^{(y, v)}(t / \lambda)\right|}{R^{1 / 2}}\right)^{-N^{10}} M\left(\mathfrak{F}_{j}^{*}\left(\rho_{v} \widehat{f}\right)\right)(y) .
$$

Therefore, we have (11).

Next we will show (2). Let

$$
\mathcal{A}_{j}^{v} f(x):=\left(c^{-2} R^{1 / 2}\right)^{-n} \int\left(1+\frac{\left|\nabla_{\xi} \Psi_{j}^{\lambda}\left(x, t_{e}, v\right)-2 \pi y\right|}{c^{-2} R^{1 / 2}}\right)^{-N^{10}}|f(y)| d y .
$$

By estimating the kernel using the stationary phase method (see Lemma 11.1), we have

$$
\left|\mathfrak{F}_{j}^{*}\left(\rho_{v} \widehat{f}\right)(x)\right| \lesssim \mathcal{A}_{j}^{v} f(x) .
$$

Moreover, since we have that

$$
\left|\nabla_{\xi} \Psi_{j}^{\lambda}\left(x, t_{e}, v\right)-\nabla_{\xi} \Psi_{j}^{\lambda}\left(x^{\prime}, t_{e}, v\right)\right| \sim\left|x-x^{\prime}\right|
$$


from (1.2), we see that if $\left|x-x^{\prime}\right| \lesssim c^{-2} R^{1 / 2}$, then

$$
\mathcal{A}_{j}^{v} f(x) \sim \mathcal{A}_{j}^{v} f\left(x^{\prime}\right) .
$$

In addition, by Schur's lemma we have

$$
\left\|\mathcal{A}_{j}^{v} f\right\|_{2} \lesssim\|f\|_{2}
$$

We may replace $\rho_{v} \widehat{f}$ with $\rho_{v}\left(\tilde{\rho}_{v}^{\vee} * f\right)^{\wedge}$, where $\tilde{\rho}_{v}$ is a bump function which is essentially supported on the $c^{2} R^{-1 / 2}$-neighborhood of the point $v$. Using (8.5) and (8.6), we have

$$
\begin{aligned}
\sum_{(y, v) \in G^{R} \times V_{j}^{R}}\left|h_{(y, v)}\right|^{2} & =R^{n / 2} \sum_{(y, v) \in G^{R} \times V_{j}^{R}}\left|M\left(\mathfrak{F}_{j}^{*}\left(\rho_{v} \widehat{f}\right)\right)(y)\right|^{2} \\
& \lesssim \sum_{(y, v) \in G^{R} \times V_{j}^{R}}\left|M\left(\mathcal{A}_{j}^{v}\left(\tilde{\rho}_{v}^{\vee} * f\right)\right)(y)\right|^{2} \\
& \lesssim \sum_{v \in V_{j}^{R}} \int\left|M\left(\mathcal{A}_{j}^{v}\left(\tilde{\rho}_{v}^{\vee} * f\right)\right)(x)\right|^{2} d x
\end{aligned}
$$

Applying the Hardy-Littlewood maximal theorem, (8.7) and the Plancherel theorem, we obtain

$$
\sum_{(y, v) \in G^{R} \times V_{j}^{R}}\left|h_{(y, v)}\right|^{2} \lesssim \sum_{v \in V_{j}^{R}} \int\left|\mathcal{A}_{j}^{v}\left(\tilde{\rho}_{v}^{\vee} * f\right)(x)\right|^{2} d x \lesssim \sum_{v} \int\left|\tilde{\rho}_{v} \widehat{f}(\xi)\right|^{2} d \xi \lesssim\|f\|_{2}^{2}
$$

Using Proposition 2.2. Lemma 8.1 and Lemma 6.2 we can obtain the following proposition, which corresponds to Proposition 3.2 .

Proposition 8.2. Let $\lambda \geq R \geq C_{0}, 0<c \leq 2^{-C_{0}}$ and let $\alpha \geq 1$ be sufficiently large. Let $Q_{R}$ be a cube of side-length $R$. Then for $p=\frac{n+3}{n+1}$, we have

$$
\left\|\mathcal{U}_{1}^{\lambda} \mathcal{U}_{2}^{\lambda}\right\|_{L^{p}\left(Q_{R}\right)} \leq(1+C N c)\left(\sum_{b \in \mathcal{Q}_{C_{0}}\left(\widetilde{Q}_{R}\right)}\left\|\mathcal{U}_{1}^{(b)} \mathcal{U}_{2}^{(b)}\right\|_{L^{p}(b)}^{p}\right)^{1 / p}+2^{C C_{0}} c^{-C}
$$

and

$$
\begin{aligned}
& \left\|\mathcal{U}_{1}^{\lambda} \mathcal{U}_{2}^{\lambda}\right\|_{L^{p}\left(Q_{R} \cap \Lambda^{\lambda}(r)\right)} \\
\leq & (1+C N c)\left(\sum_{b \in \mathcal{Q}_{C_{0}}\left(\widetilde{Q}_{R}\right)}\left\|\mathcal{U}_{1}^{(b)} \mathcal{U}_{2}^{(b)}\right\|_{L^{p}\left(b \cap \Lambda^{\lambda}(r)\right)}^{p}\right)^{1 / p}+2^{C C_{0}} c^{-C}\left(\frac{R}{r}\right)^{-\frac{n-1}{2(n+3)}} .
\end{aligned}
$$

Here, $\widetilde{Q}_{R}$ is a cube of side-length $C R$ and is contained in $C^{2} Q_{R}$. The $\mathcal{U}_{1}^{(b)}, \mathcal{U}_{2}^{(b)}$ stand for $\mathcal{U}_{1}^{(b), \mathcal{U}_{2}}, \mathcal{U}_{2}^{(b),\left[\mathcal{U}_{1}^{\mathcal{U}_{2}}\right]_{C_{0}}}$, respectively.

We skip the proof of this proposition, because its proof is almost the same as that of Proposition 3.2. Following the argument of Proposition 3.2, we just substitute Lemma 8.1 for Lemma 3.1 and it is possible to apply Proposition 2.2 by (6.2). 


\section{IMPROVED RECURSIVE INEQUALITY}

We will apply the induction-on-scale argument to the energy-concentrated area $\Omega^{e}=Q_{R}^{e} \cap \Lambda^{\lambda, z_{e}}\left(C_{0}\left(r_{e}+1\right)\right)$. For this, we state a new induction hypothesis.

Hypothesis 9.1. Let $\lambda \geq R \geq 2^{C_{0}}, r>0$ and $r^{\prime}>0$. Let $\Lambda^{\lambda}=\Lambda_{1}^{\lambda} \cup \Lambda_{2}^{\lambda}$ with vertex $z_{e}=\left(x_{e}, t_{e}\right)$, and let $\mathcal{U}_{j}^{\lambda}=\mathcal{U}_{j}^{\lambda,\left(t_{e}, \alpha\right)}$. We define $A^{\lambda}\left(R, r, r^{\prime}\right)$ as the least constant such that the inequality

$$
\left\|\mathcal{U}_{1}^{\lambda} \mathcal{U}_{2}^{\lambda}\right\|_{L^{p}\left(Q_{R} \cap \Lambda^{\lambda}\left(r^{\prime}\right)\right)} \leq A^{\lambda}\left(R, r, r^{\prime}\right)\left(E\left(\mathcal{U}_{1}^{\lambda}\right)^{1 / 2} E\left(\mathcal{U}_{2}^{\lambda}\right)^{1 / 2}\right)^{1 / p} E_{r}\left(\mathcal{U}_{1}^{\lambda}, \mathcal{U}_{2}^{\lambda}\right)^{1 / p^{\prime}}
$$

holds for any cube $Q_{R}$ of side-length $R$. Here, we define $E_{r}\left(\mathcal{U}_{1}^{\lambda}, \mathcal{U}_{2}^{\lambda}\right)$ as

$$
E_{r}\left(\mathcal{U}_{1}^{\lambda}, \mathcal{U}_{2}^{\lambda}\right)=\max \left(\frac{1}{2} E\left(\mathcal{U}_{1}^{\lambda}\right)^{1 / 2} E\left(\mathcal{U}_{2}^{\lambda}\right)^{1 / 2},\left\|U_{1}^{\lambda}(\cdot ; \alpha)\right\|_{L^{2}(D)}\left\|U_{j}^{\lambda}(\cdot ; \alpha)\right\|_{L^{2}(D)}\right),
$$

where $D$ is the disc of radius $r$ and of center $z_{e}$.

Lemma 9.2. Let $2^{C_{0}} \leq R \leq \lambda$ and let $V_{j}^{R}\left(\xi_{0}\right):=\Xi_{j} \cap\left(R^{-1 / 2} \mathbb{Z}^{n}+\xi_{0}\right)$, where $R^{-1 / 2} \mathbb{Z}^{n}+\xi_{0}$ denotes the $\xi_{0}$-translation of $R^{-1 / 2} \mathbb{Z}^{n}$. Let $q_{0}$ be the cube of sidelength $C R^{-1 / 2}$ and centered at the origin and let $U_{j}^{(b)}$ be defined as in (3.2) but with $\mathcal{T}_{j}^{R}=G^{R} \times V_{j}^{R}\left(\xi_{0}\right)$. Then for a sufficiently large $\alpha \geq 1$ and some $\xi_{0} \in q_{0}$, we have

$$
\left(\sum_{b \in \mathcal{Q}_{C_{0}}\left(\widetilde{Q}_{R}\right)}\left\|U_{j}^{(b)}\left(t_{e} ; \alpha\right)\right\|_{2}^{2}\right)^{1 / 2} \leq(1+C c)\left\|U_{j}^{\lambda}\left(t_{e} ; \alpha\right)\right\|_{2} .
$$

Proof. The left side of (9.1) is written as

$$
\left(\sum_{b \in \mathcal{Q}_{C_{0}}\left(\widetilde{Q}_{R}\right)}\left\|\sum_{v \in V_{j}^{R}} \sum_{y \in G^{R}} m_{(y, v)}^{(b)} a_{\times}^{\alpha \lambda} \eta_{y} \mathfrak{F}_{j}^{*}\left(\rho_{v} \widehat{f}\right)\right\|_{2}^{2}\right)^{1 / 2} .
$$

As in the proof of Lemma 5.4 it suffices to show that for some $\xi_{0} \in q_{0}$ we have

$$
\left(\sum_{b \in \mathcal{Q}_{C_{0}}\left(\widetilde{Q}_{R}\right)}\left\|\sum_{v \in V_{j}^{R}} \sum_{y \in G^{R}} m_{(y, v)}^{(b)} a_{\times}^{\alpha \lambda} \eta_{y} \mathfrak{F}_{j}^{*}\left(\chi_{W_{j}^{R}\left(\xi_{0}\right)} \rho_{v} \widehat{f}\right)\right\|_{2}^{2}\right)^{1 / 2} \leq(1+c)\left\|U_{j}^{\lambda}\left(t_{e} ; \alpha\right)\right\|_{2},
$$

$$
\left(\sum_{b \in \mathcal{Q}_{C_{0}}\left(\widetilde{Q}_{R}\right)}\left\|\sum_{v \in V_{j}^{R}} \sum_{y \in G^{R}} m_{(y, v)}^{(b)} a_{\times}^{\alpha \lambda} \eta_{y} \mathfrak{F}_{j}^{*}\left(\left(1-\chi_{W_{j}^{R}\left(\xi_{0}\right)}\right) \rho_{v} \hat{f}\right)\right\|_{2}^{2}\right)^{1 / 2} \leq C c\left\|U_{j}^{\lambda}\left(t_{e} ; \alpha\right)\right\|_{2}
$$

where $W_{j}^{R}\left(\xi_{0}\right)$ is defined in (5.4).

The difference from Lemma 5.4 is that we use $\mathfrak{F}_{j}^{*}$ instead of the Fourier transform $\mathcal{F}^{-1}$. Thus, it is necessary to show the following orthogonality and almost orthogonality. For any $v, v^{\prime} \in V_{j}^{\lambda}$ and $v \neq v^{\prime}$, we have

$$
\left\langle\eta_{y} \mathfrak{F}_{j}^{*}\left(\chi_{W_{j}^{R}\left(\xi_{0}\right)} \rho_{v} f\right), \eta_{y} \mathfrak{F}_{j}^{*}\left(\chi_{W_{j}^{R}\left(\xi_{0}\right)} \rho_{v^{\prime}} f\right)\right\rangle<\epsilon, \quad \forall \epsilon>0,
$$

$$
\left\langle\eta_{y} \mathfrak{F}_{j}^{*}\left(\left(1-\chi_{W_{j}^{R}\left(\xi_{0}\right)}\right) \rho_{v} f\right), \eta_{y} \mathfrak{F}_{j}^{*}\left(\left(1-\chi_{W_{j}^{R}\left(\xi_{0}\right)}\right) \rho_{v^{\prime}} f\right)\right\rangle \lesssim \frac{1}{\left(1+C R^{1 / 2}\left|v-v^{\prime}\right|\right)^{N^{10}}}
$$


Consider (9.4). The left side of (9.4) is written as

$$
\iint K(\xi, \zeta)\left(\chi_{W_{j}^{R}\left(\xi_{0}\right)} \rho_{v} f\right)(\xi) \overline{\left(\chi_{W_{j}^{R}\left(\xi_{0}\right)} \rho_{v^{\prime}} f\right)(\zeta)} d \xi d \zeta
$$

where

$$
K(\xi, \zeta)=a_{\Xi_{j}}(\xi) a_{\Xi_{j}}(\zeta) \int e^{i\left(\psi_{j}^{\lambda}\left(x, t_{e}, \xi\right)-\psi_{j}^{\lambda}\left(x, t_{e}, \zeta\right)\right)} \eta_{y}^{2}(x) d x
$$

By (1.2) and the stationary phase method (see Lemma 11.1), we have

$$
|K(\xi, \zeta)| \lesssim\left(c^{-2} R^{1 / 2}\right)^{n}\left(1+c^{-2} R^{1 / 2}|\xi-\zeta|\right)^{-N^{10}} .
$$

We divide (9.6) into two parts. (9.6) is bounded by

$$
\begin{aligned}
\iint_{|\xi-\zeta|<c^{2} R^{-1 / 2}}|K(\xi, \zeta)|\left|\left(\chi_{W_{j}^{R}\left(\xi_{0}\right)} \rho_{v} f\right)(\xi) \overline{\left(\chi_{W_{j}^{R}\left(\xi_{0}\right)} \rho_{v^{\prime}} f\right)(\zeta)}\right| d \xi d \zeta \\
\quad+\iint_{|\xi-\zeta|>c^{2} R^{-1 / 2}}|K(\xi, \zeta)|\left|\left(\chi_{W_{j}^{R}\left(\xi_{0}\right)} \rho_{v} f\right)(\xi) \overline{\left(\chi_{W_{j}^{R}\left(\xi_{0}\right)} \rho_{v^{\prime}} f\right)(\zeta)}\right| d \xi d \zeta .
\end{aligned}
$$

The first integral vanishes because $\left(\chi_{W_{j}^{R}\left(\xi_{0}\right)} \rho_{v} f\right)(\xi) \overline{\left(\chi_{W_{j}^{R}\left(\xi_{0}\right)} \rho_{v^{\prime}} f\right)(\zeta)}$ is zero provided $|\xi-\zeta|<C c^{2} R^{-1 / 2}$. The other integral is arbitrarily small, because by Young's inequality and (1.11), it is bounded by

$$
\left(c^{-2} R^{1 / 2}\right)^{n} \int_{|\xi|>c^{2} R^{-1 / 2}}\left(1+c^{-2} R^{1 / 2}|\xi|\right)^{-N^{10}} d \xi \leq 2^{-\left(N^{10}+1\right)} .
$$

Hence, we obtain (9.4).

Consider (9.5). We can observe that if $\left|v-v^{\prime}\right| \gtrsim R^{-1 / 2}$, then

$$
\left(\left(1-\chi_{W_{j}^{R}\left(\xi_{0}\right)}\right) \rho_{v} f\right)(\xi) \overline{\left(\left(1-\chi_{W_{j}^{R}\left(\xi_{0}\right)}\right) \rho_{v^{\prime}} f\right)(\zeta)}
$$

almost vanishes; i.e., its value is extremely small. So we have that

$$
\begin{aligned}
& \iint|K(\xi, \zeta)|\left|\left(\left(1-\chi_{W_{j}^{R}\left(\xi_{0}\right)}\right) \rho_{v} f\right)(\xi) \overline{\left(\left(1-\chi_{W_{j}^{R}\left(\xi_{0}\right)}\right) \rho_{v^{\prime}} f\right)(\zeta)}\right| d \xi d \zeta \\
& \lesssim \frac{1}{\left(1+C^{-1} R^{1 / 2}\left|v-v^{\prime}\right|\right)^{N^{10}}}
\end{aligned}
$$

which implies (9.5).

As in the proof of Lemma 5.4 using (9.4) and (9.5) we can show that for some $\xi_{0} \in q_{0}$, the left sides of (9.2) and (9.3) are bounded by

$$
(1+c)\left\|a_{\times}^{\alpha \lambda} \mathfrak{F}_{j}^{*}\left(\chi_{W_{j}^{R}\left(\xi_{0}\right)} \hat{f}\right)\right\|_{2} \quad \text { and } \quad C c\left\|a_{\times}^{\alpha \lambda} \mathfrak{F}_{j}^{*}\left(\left(1-\chi_{W_{j}^{R}\left(\xi_{0}\right)}\right) \widehat{f}\right)\right\|_{2},
$$

respectively.

Lastly, to obtain (9.4) and (9.5) we will show that

$$
\left\|a_{\times}^{\alpha \lambda} \mathfrak{F}_{j}^{*}\left(\chi_{W_{j}^{R}\left(\xi_{0}\right)} \hat{f}\right)\right\|_{2}, \quad\left\|a_{\times}^{\alpha \lambda} \mathfrak{F}_{j}^{*}\left(\left(1-\chi_{W_{j}^{R}\left(\xi_{0}\right)}\right) \widehat{f}\right)\right\|_{2} \leq(1+c)\left\|U_{j}^{\lambda}\left(t_{e} ; \alpha\right)\right\|_{2} .
$$

Since $U_{j}^{\lambda}\left(t_{e} ; \alpha\right)=a_{\times}^{\alpha \lambda} \mathfrak{F}_{j}^{*}\left(\chi_{W_{j}^{R}\left(\xi_{0}\right)} \hat{f}\right)+a_{\times}^{\alpha \lambda} \mathfrak{F}_{j}^{*}\left(\left(1-\chi_{W_{j}^{R}\left(\xi_{0}\right)}\right) \widehat{f}\right)$, it suffices to show that

$$
\left\langle a_{\times}^{\alpha \lambda} \mathfrak{F}_{j}^{*}\left(\chi_{W_{j}^{R}\left(\xi_{0}\right)} \widehat{f}\right), a_{\times}^{\alpha \lambda} \mathfrak{F}_{j}^{*}\left(\left(1-\chi_{W_{j}^{R}\left(\xi_{0}\right)}\right) \widehat{f}\right)\right\rangle<c .
$$

We write the left side of (9.7) as

$$
\left\langle\mathfrak{F}_{j}\left(a_{\times}^{\alpha \lambda}\right)^{2} \mathfrak{F}_{j}^{*}\left(\chi_{W_{j}^{R}\left(\xi_{0}\right)} \hat{f}\right),\left(1-\chi_{W_{j}^{R}\left(\xi_{0}\right)}\right) \hat{f}\right\rangle .
$$


Since $\left\langle\chi_{W_{j}^{R}\left(\xi_{0}\right)} \widehat{f},\left(1-\chi_{W_{j}^{R}\left(\xi_{0}\right)}\right) \widehat{f}\right\rangle=0$, it is equal to

$$
\left\langle\mathfrak{F}_{j}\left(a_{\times}^{\alpha \lambda}\right)^{2} \mathfrak{F}_{j}^{*}\left(\chi_{W_{j}^{R}\left(\xi_{0}\right)} \widehat{f}\right)-\chi_{W_{j}^{R}\left(\xi_{0}\right)} \widehat{f},\left(1-\chi_{W_{j}^{R}\left(\xi_{0}\right)}\right) \widehat{f}\right\rangle .
$$

By the Cauchy-Schwarz inequality and (1.11), it is bounded by

$$
\left\|\mathfrak{F}_{j}\left(a_{\times}^{\alpha \lambda}\right)^{2} \mathfrak{F}_{j}^{*}\left(\chi_{W_{j}^{R}\left(\xi_{0}\right)} \widehat{f}\right)-\chi_{W_{j}^{R}\left(\xi_{0}\right)} \widehat{f}\right\|_{2} \cdot
$$

From Lemma 6.1 and (8.1), we see that

$$
\mathfrak{F}_{j}\left(a_{\times}^{\alpha \lambda}\right)^{2} \mathfrak{F}_{j}^{*}\left(\chi_{W_{j}^{R}\left(\xi_{0}\right)} \widehat{f}\right)
$$

converges to $\chi_{W_{j}^{R}\left(\xi_{0}\right)} \widehat{f}$ in $L^{2}$ as $\alpha \rightarrow \infty$. Therefore, if $\alpha>1$ is sufficiently large, then we obtain (9.7).

Now we apply the induction-on-scale argument to $\left\|\mathcal{U}_{1}^{\lambda} \mathcal{U}_{1}^{\lambda}\right\|_{L^{p}\left(Q_{R} \cap \Lambda^{\lambda}\left(r^{\prime}\right)\right)}$.

Proposition 9.3. Let $\lambda \geq R \geq 2^{C_{0}}, R^{1 / 2+3 / N}<r<C_{0}^{C} R$ and $r^{\prime}>0$. Then for any $0<c \leq 2^{-C_{0}}$, we have

$$
A^{\lambda}\left(R, r, r^{\prime}\right) \leq(1+C N c) A^{\lambda}\left(2^{-C_{0}} R, r, r^{\prime}\right)+2^{C C_{0}} c^{-C}(R / r)^{-\tau},
$$

where $\tau=\frac{n-1}{2(n+3)}$.

Proof. By Hypothesis 9.1, it suffices to show that

$$
\begin{aligned}
& \left\|\mathcal{U}_{1}^{\lambda} \mathcal{U}_{2}^{\lambda}\right\|_{L^{p}\left(Q_{R} \cap \Lambda^{\lambda}\left(r^{\prime}\right)\right)} \\
& \leq(1+C N c) A^{\lambda}\left(2^{-C_{0}} R, r, r^{\prime}\right)\left(\left\|U_{1}^{\lambda}\left(t_{e} ; \alpha\right)\right\|_{2}\left\|U_{2}^{\lambda}\left(t_{e} ; \alpha\right)\right\|_{2}\right)^{1 / p} E_{r}\left(\mathcal{U}_{1}^{\lambda}, \mathcal{U}_{2}^{\lambda}\right)^{1 / p^{\prime}} \\
& +2^{C C_{0}} c^{-C}(R / r)^{-\tau} .
\end{aligned}
$$

The proof is similar to that of Proposition 5.2. By Lemma 4.1 and Proposition 3.2 , we have

$$
\begin{aligned}
& \left\|\mathcal{U}_{1}^{\lambda} \mathcal{U}_{2}^{\lambda}\right\|_{L^{p}\left(Q_{R} \cap \Lambda^{\lambda}\left(r^{\prime}\right)\right)} \\
& \quad \leq(1+C N c)\left(\sum_{b \in \mathcal{Q}_{C_{0}}\left(\widetilde{Q}_{R}\right)}\left\|\mathcal{U}_{1}^{(b)} \mathcal{U}_{2}^{(b)}\right\|_{L^{p}\left(b \cap \Lambda^{\lambda}\left(r^{\prime}\right)\right)}^{p}\right)^{1 / p}+2^{C C_{0}} c^{-C}(R / r)^{-\tau},
\end{aligned}
$$

where $\widetilde{Q}_{R}$ is a cube of side-length $C R$ which is contained in $C^{2} Q_{R}$. So, it is enough to show that

$$
\begin{aligned}
& \left(\sum_{b \in \mathcal{Q}_{C_{0}}\left(\widetilde{Q}_{R}\right)}\left\|\mathcal{U}_{1}^{(b)} \mathcal{U}_{2}^{(b)}\right\|_{L^{p}\left(b \cap \Lambda^{\lambda}\left(r^{\prime}\right)\right)}^{p}\right)^{1 / p} \\
& \leq(1+C c) A^{\lambda}\left(2^{-C_{0}} R, r, r^{\prime}\right)\left(\left\|U_{1}^{\lambda}\left(t_{e} ; \alpha\right)\right\|_{2}\left\|U_{2}^{\lambda}\left(t_{e} ; \alpha\right)\right\|_{2}\right)^{1 / p} E_{r}\left(\mathcal{U}_{1}^{\lambda}, \mathcal{U}_{2}^{\lambda}\right)^{1 / p^{\prime}} .
\end{aligned}
$$

By Hypothesis 9.1 we have

$$
\begin{aligned}
\| \mathcal{U}_{1}^{(b)} \mathcal{U}_{2}^{(b)} & \|_{L^{p}\left(b \cap \Lambda^{\lambda}\left(r^{\prime}\right)\right)} \\
& \leq A^{\lambda}\left(2^{-C_{0}} R, r, r^{\prime}\right)\left(\left\|U_{1}^{(b)}\left(t_{e} ; \alpha\right)\right\|_{2}\left\|U_{2}^{(b)}\left(t_{e} ; \alpha\right)\right\|_{2}\right)^{1 / p} E_{r}\left(\mathcal{U}_{1}^{(b)}, \mathcal{U}_{2}^{(b)}\right)^{1 / p^{\prime}} .
\end{aligned}
$$

From Lemma 9.2 we easily get

$$
E_{r}\left(\mathcal{U}_{1}^{(b)}, \mathcal{U}_{2}^{(b)}\right) \leq(1+C c) E_{r}\left(\mathcal{U}_{1}^{\lambda}, \mathcal{U}_{2}^{\lambda}\right)
$$


because

$$
\begin{aligned}
\left\|U_{j}^{(b)}\left(t_{e} ; \alpha\right)\right\|_{2} & \leq(1+C c)\left\|U_{j}^{\lambda}\left(t_{e} ; \alpha\right)\right\|_{2}, \\
\left\|U_{j}^{(b)}(\cdot ; \alpha)\right\|_{L^{2}(D)} & \leq(1+C c)\left\|U_{j}^{\lambda}(\cdot ; \alpha)\right\|_{L^{2}(D)} .
\end{aligned}
$$

Thus, to obtain (9.8) it suffices to show that

$$
\sum_{b \in \mathcal{Q}_{C_{0}}\left(\widetilde{Q}_{R}\right)}\left\|U_{1}^{(b)}\left(t_{e} ; \alpha\right)\right\|_{2}\left\|U_{2}^{(b)}\left(t_{e} ; \alpha\right)\right\|_{2} \leq(1+C c)\left\|U_{1}^{\lambda}\left(t_{e} ; \alpha\right)\right\|_{2}\left\|U_{2}^{\lambda}\left(t_{e} ; \alpha\right)\right\|_{2} .
$$

This follows from the Cauchy-Schwarz inequality and Lemma 9.2 .

By iterating the new recursive inequality, we have the following corollary.

Corollary 9.4. Let $R \geq 2^{C_{0}}$ and $r \geq R^{1 / 2+4 / N}$. Suppose that $J$ is the integer such that $2^{-J} C_{0}^{C} R \leq r \leq 2^{-J+1} C_{0}^{C} R$. Then for any $0<c \leq 2^{-C_{0}}$, we have

$$
\overline{A^{\lambda}}\left(R, r, C_{0}(1+r)\right) \leq(1+C c) \overline{A^{\lambda}}\left(2^{-J} R, r, C_{0}(1+r)\right)+C c^{-C},
$$

where

$$
\overline{A^{\lambda}}\left(R, r, r^{\prime}\right)=\sup _{2^{C_{0}} \leq R^{\prime} \leq R} A^{\lambda}\left(R^{\prime}, r, r^{\prime}\right) .
$$

Proof. From Proposition 9.3, we have

$$
\overline{A^{\lambda}}\left(R / 2^{j}, r, C_{0}(1+r)\right) \leq\left(1+C c_{j}\right) \overline{A^{\lambda}}\left(R / 2^{j+1}, r, C_{0}(1+r)\right)+C c_{j}^{-C} 2^{-(J-j) \tau} .
$$

By iterating this recursive inequality, we have

$$
\overline{A^{\lambda}}\left(R, r, C_{0}(1+r)\right) \leq\left(\prod_{j=0}^{J-1}\left(1+C c_{j}\right)\right)\left(\overline{A^{\lambda}}\left(2^{-J} R, r, C_{0}(1+r)\right)+\sum_{j=0}^{J-1} c_{j}^{-C} 2^{-(J-j) \tau}\right) .
$$

If we set $c_{j}=c 2^{-(J-j) \tau /(2 C)}$ for a suitable constant $C$, then we have

$$
\prod_{j=0}^{J-1}\left(1+C c_{j}\right) \leq \exp \left(C\left(c_{0}+\cdots+c_{J-1}\right)\right) \leq \exp (C c) \leq 1+C^{2} c
$$

and

$$
\sum_{j=0}^{J-1} c_{j} 2^{(J-j) \gamma} \leq C c^{-C}
$$

Thus, we obtain

$$
\overline{A^{\lambda}}\left(R, r, C_{0}(1+r)\right) \leq(1+C c) \overline{A^{\lambda}}\left(2^{-J} R, r, C_{0}(1+r)\right)+C c^{-C} .
$$

Proposition 9.5. Let $\lambda \geq R \geq 2^{C_{0}}, r \geq C_{0} R, r^{\prime}>0$ and $0<c \leq 2^{-C_{0}}$. Then we have

$$
A^{\lambda}\left(R, r, r^{\prime}\right) \leq A^{\lambda}(R)+2^{C C_{0}} .
$$

Proof. We have to show that

$$
\begin{aligned}
& \left\|\mathcal{U}_{1}^{\lambda} \mathcal{U}_{2}^{\lambda}\right\|_{L^{p}\left(Q_{R} \cap \Lambda^{\left.\lambda, z_{e}\left(r^{\prime}\right)\right)}\right.} \\
& \leq A^{\lambda}(R)\left(E\left(\mathcal{U}_{1}^{\lambda}\right)^{1 / 2} E\left(\mathcal{U}_{2}^{\lambda}\right)^{1 / 2}\right)^{1 / p} E_{r}\left(\mathcal{U}_{1}^{\lambda}, \mathcal{U}_{2}^{\lambda}\right)^{1 / p^{\prime}}+2^{C C_{0}}
\end{aligned}
$$


Let $D$ be a disk of radius $r / 2$ and centered at $z_{e}$. Using Lemma 6.4 and Hölder's inequality, we have

$$
\begin{aligned}
\left\|\left(1-L_{D}\right) \mathcal{U}_{1}^{\lambda} \mathcal{U}_{2}^{\lambda}\right\|_{L^{p}\left(Q_{R}\right)} & \lesssim R^{-N+C}, \\
\left\|L_{D} \mathcal{U}_{1}^{\lambda}\left(1-L_{D}\right) \mathcal{U}_{2}^{\lambda}\right\|_{L^{p}\left(Q_{R}\right)} & \lesssim R^{-N+C} .
\end{aligned}
$$

So, by the triangle inequality it suffices to show that

$$
\begin{aligned}
\left\|\left(L_{D} \mathcal{U}_{1}^{\lambda}\right)\left(L_{D} \mathcal{U}_{2}^{\lambda}\right)\right\|_{L^{p}\left(Q_{R}\right)} \\
\quad \leq A^{\lambda}(R)\left(E\left(\mathcal{U}_{1}^{\lambda}\right)^{1 / 2} E\left(\mathcal{U}_{2}^{\lambda}\right)^{1 / 2}\right)^{1 / p} E_{r}\left(\mathcal{U}_{1}^{\lambda}, \mathcal{U}_{2}^{\lambda}\right)^{1 / p^{\prime}}+2^{C C_{0}} .
\end{aligned}
$$

By Hypothesis 7.2, we have

$$
\left\|\left(L_{D} \mathcal{U}_{1}^{\lambda}\right)\left(L_{D} \mathcal{U}_{2}^{\lambda}\right)\right\|_{L^{p}\left(Q_{R}\right)} \leq A^{\lambda}(R) E\left(L_{D} \mathcal{U}_{1}^{\lambda}\right)^{1 / 2} E\left(L_{D} \mathcal{U}_{2}^{\lambda}\right)^{1 / 2}
$$

The pointwise estimates give

$$
E\left(L_{D} \mathcal{U}_{j}^{\lambda}\right)=\left\|\left(\chi_{D} * \eta_{r^{1-1 / N}}\right) U_{j}^{\lambda}\left(t_{e} ; \alpha\right)\right\|_{2}^{2} \leq\left\|U_{j}^{\lambda}(\cdot ; \alpha)\right\|_{L^{2}(D)}^{2}+C R^{-N+C} .
$$

Thus we have

$$
\begin{aligned}
E\left(L_{D} \mathcal{U}_{1}^{\lambda}\right)^{1 / 2} E\left(L_{D} \mathcal{U}_{2}^{\lambda}\right)^{1 / 2} & \leq\left\|U_{1}^{\lambda}(\cdot ; \alpha)\right\|_{L^{2}(D)}\left\|U_{2}^{\lambda}(\cdot ; \alpha)\right\|_{L^{2}(D)}+C R^{-N+C} \\
& \leq E_{r}\left(\mathcal{U}_{1}^{\lambda}, \mathcal{U}_{2}^{\lambda}\right)+C R^{-N+C} .
\end{aligned}
$$

Inserting this into (9.11), we obtain that

$$
\begin{aligned}
& \left\|\left(L_{D} \mathcal{U}_{1}^{\lambda}\right)\left(L_{D} \mathcal{U}_{2}^{\lambda}\right)\right\|_{L^{p}\left(Q_{R}\right)} \\
& \quad \leq A^{\lambda}(R)\left(E\left(\mathcal{U}_{1}^{\lambda}\right)^{1 / 2} E\left(\mathcal{U}_{2}^{\lambda}\right)^{1 / 2}\right)^{1 / p}\left(E_{r}\left(\mathcal{U}_{1}^{\lambda}, \mathcal{U}_{2}^{\lambda}\right)+C R^{-N+C}\right)^{1 / p^{\prime}} .
\end{aligned}
$$

Since we already have the trivial estimate $A^{\lambda}(R) \lesssim A(R) \lesssim R^{C}$, this yields (9.10).

\section{Proof of Theorem 1.1}

By the triangle inequality we have

$$
\left\|U_{1}^{\lambda} U_{2}^{\lambda}\right\|_{p} \leq\left\|\mathcal{U}_{1}^{\lambda} \mathcal{U}_{2}^{\lambda}\right\|_{p}+\left\|\mathcal{G}_{1}^{\lambda} f \mathcal{G}_{2}^{\lambda}\left(g-g_{\left(t_{e} ; \alpha\right)}\right)\right\|_{p}+\left\|\mathcal{G}_{1}^{\lambda}\left(f-f_{\left(t_{e} ; \alpha\right)}\right) \mathcal{G}_{2}^{\lambda} g_{\left(t_{e} ; \alpha\right)}\right\|_{p}
$$

If we take $\alpha \geq 1$ such that

$$
\left\|f-f_{\alpha}\right\|_{2} \leq 1 / 4 \quad \text { and } \quad\left\|g-g_{\alpha}\right\|_{2} \leq 1 / 4,
$$

then by Hypothesis 5.1 we have

$$
\left\|\mathcal{G}_{1}^{\lambda} f \mathcal{G}_{2}^{\lambda}\left(g-g_{\left(t_{e} ; \alpha\right)}\right)\right\|_{p} \leq \frac{A(\lambda)}{4} \quad \text { and } \quad\left\|\mathcal{G}_{1}^{\lambda}\left(f-f_{\left(t_{e} ; \alpha\right)}\right) \mathcal{G}_{2}^{\lambda} g_{\left(t_{e} ; \alpha\right)}\right\|_{p} \leq \frac{A(\lambda)}{4} .
$$

So, we have

$$
A(\lambda) \leq 2\left\|\mathcal{U}_{1}^{\lambda} \mathcal{U}_{2}^{\lambda}\right\|_{p}
$$

Therefore, it suffices to show that

$$
\left\|\mathcal{U}_{1}^{\lambda} \mathcal{U}_{2}^{\lambda}\right\|_{p} \lesssim 2^{C C_{0}} .
$$

We may assume that, in the extremal case,

$$
\left\|\mathcal{U}_{1}^{\lambda} \mathcal{U}_{2}^{\lambda}\right\|_{p} \sim A^{\lambda}(\lambda)
$$

By Corollary 7.7, we have

$$
\left\|\mathcal{U}_{1}^{\lambda} \mathcal{U}_{2}^{\lambda}\right\|_{p} \leq\left(1-C\left(\delta+C_{0}^{-C}\right)^{p}\right)^{-1 / p}\left\|\mathcal{U}_{1}^{\lambda} \mathcal{U}_{2}^{\lambda}\right\|_{L^{p}\left(Q_{R}^{e} \cap \Lambda^{\lambda, z_{e}}\left(C_{0} r_{e}+C_{0}\right)\right)} .
$$


By combining Corollary 9.4 and Proposition 9.5, we have

$$
\begin{aligned}
& \left\|\mathcal{U}_{1}^{\lambda} \mathcal{U}_{2}^{\lambda}\right\|_{L^{p}\left(Q_{R}^{e} \cap \Lambda^{\lambda, z_{e}}\left(C_{0} r_{e}+C_{0}\right)\right)} \\
& \quad \leq(1+C N c)\left(\overline{A^{\lambda}}(R)+2^{C C_{0}}\right)\left(E\left(\mathcal{U}_{1}^{\lambda}\right)^{1 / 2} E\left(\mathcal{U}_{2}^{\lambda}\right)^{1 / 2}\right)^{1 / p} E_{r}\left(\mathcal{U}_{1}^{\lambda}, \mathcal{U}_{2}^{\lambda}\right)^{1 / p^{\prime}} .
\end{aligned}
$$

By (7.1), we have

$$
E_{r}\left(\mathcal{U}_{1}^{\lambda}, \mathcal{U}_{2}^{\lambda}\right) \leq(1-\delta)\left\|U_{1}^{\lambda}\left(t_{e} ; \alpha\right)\right\|_{2}\left\|U_{2}^{\lambda}\left(t_{e} ; \alpha\right)\right\|_{2} .
$$

Thus, we obtain that

$$
A^{\lambda}(\lambda) \leq(1+C N c)\left(1-C\left(\delta+C_{0}^{-C}\right)^{p}\right)^{-1 / p}(1-\delta)^{1 / p^{\prime}} \overline{A^{\lambda}}(R)+2^{C C_{0}} .
$$

If we take $\delta=C_{0}^{-C}$ for some appropriate $C$, then $\left(1-C\left(\delta+C_{0}^{-C}\right)^{p}\right)^{-1 / p}(1-\delta)^{1 / p^{\prime}}$ is strictly less than 1 . If we take $c$ to be a very small number such as $c=2^{-C C_{0}}$, then $\left(1-C\left(\delta+C_{0}^{-C}\right)^{p}\right)^{-1 / p}(1-\delta)^{1 / p^{\prime}}(1+C c)$ is also less than 1 . Thus, we have

$$
A^{\lambda}(\lambda) \leq\left(1-C_{0}^{-C}\right) \overline{A^{\lambda}}(R)+2^{C C_{0}} .
$$

From this we have

$$
\overline{A^{\lambda}}(\lambda) \leq\left(1-C_{0}^{-C}\right) \overline{A^{\lambda}}(\lambda)+2^{C C_{0}},
$$

and we obtain $A^{\lambda}(\lambda) \lesssim 2^{C C_{0}}$, which implies (10.1).

\section{Appendix: Stationary phase method}

In this section, we state a stationary phase method, which is used repeatedly in the paper. Let

$$
K(x)=\int e^{i \Phi(x, \xi)} a(x, \xi) d \xi
$$

where $\Phi$ is a smooth real-valued function and $a$ is a compactly supported smooth function.

Lemma 11.1 (Stationary phase method). Letting $u(x, \xi)$ be a nonvanishing vectorvalued smooth function, we set

$$
\Delta(x, \xi):=\left\langle u(x, \xi), \nabla_{\xi} \Phi(x, \xi)\right\rangle .
$$

Suppose that we have a function $\delta(x)$ such that

$$
|\Delta(x, \xi)| \geq|\delta(x)|
$$

on the support of $a$. Then we have

$$
|K(x)| \leq C(1+|\delta(x)|)^{-N}, \quad \forall N \geq 0 .
$$

Proof. Since $a$ has compact support, we have the trivial bound

$$
|K(x)| \leq C .
$$

Suppose $\delta$ is nonzero. Then we can set

$$
D_{\xi}:=[i \Delta]^{-1}\left\langle u, \nabla_{\xi}\right\rangle \text {. }
$$

Since we have

$$
D_{\xi} e^{i \Phi(z, \xi)}=e^{i \Phi(x, t, \xi)},
$$

applying the integration by parts $N$ times to the integral $K$, we obtain

$$
K(x)=\int e^{i \lambda \Phi(x, \xi)}\left(D_{\xi}^{*}\right)^{N} a(x, \xi) d \xi .
$$


Therefore, from (11.1) we have

$$
|K(x)| \leq C|\delta(x)|^{-N} .
$$

Combine this and (11.3). Then we obtain (11.2).

By this lemma, we see that on the set $\{x:|\delta(x)| \geq 1\}$, the value of the function $K$ is almost zero; i.e., the essential support of $K$ is contained in $\{x:|\delta(x)| \ll 1\}$.

Consider the case that the $\xi$-support of $a$ is contained in some neighborhood of $\xi_{0}$ and $\nabla_{\xi} \Phi(x, \xi)$ is nonzero on the support of $a$ except at $\xi=\xi_{0}$. If we take $u=\nabla_{\xi} \Phi /\left|\nabla_{\xi} \Phi\right|$ for $\xi \neq \xi_{0}$ and $\delta(x)=\nabla_{\xi} \Phi\left(x, \xi_{0}\right)$, then we have

$$
|K(x)| \lesssim\left(1+\left|\nabla_{\xi} \Phi\left(x, \xi_{0}\right)\right|\right)^{-N} .
$$

This shows that $K$ is essentially supported on some neighborhood of the stationary point $\left\{x: \nabla_{\xi} \Phi\left(x, \xi_{0}\right)=0\right\}$.

\section{ACKNOWLEDGMENTS}

The author would like to express deep gratitude to his supervisor, Jong-Guk Bak, for his guidance and support and thank Sanghyuk Lee for several useful discussions and comments. This work was done during his Ph.D. studies at the Pohang University of Science and Technology.

\section{REFERENCES}

1. B. Barcelo, On the restriction of the Fourier transform to a conical surface, Trans. Amer. Math. Soc. 292 (1985), 321-333. MR.805965 (86k:42023)

2. J. Bourgain, Besicovitch type maximal operators and applications to Fourier analysis, Geom. Funct. Anal. 1 (1991), 147-187. MR.1097257 (92g:42010)

3. J. Bourgain, Estimates for cone multipliers, in Geometric Aspects of Functional Analysis, Oper. Theory Adv. Appl., vol. 77, Birkhäuser, Basel, 1995, pp. 41-60. MR.1353448 (96m:42022)

4. J. Bourgain, Some new estimates on oscillatory integrals, Essays on Fourier Analysis in Honor of Elias M. Stein, vol. 42, Princeton Univ. Press, Princeton, NJ, 1995, pp. 83-112. MR.1315543 (96c:42028)

5. L. Carleson and P. Sjölin, Oscillatory integrals and a multiplier problem for the disc, Studia Math. 44 (1972), 287-299. MR0361607 (50:14052)

6. L. Hörmander, Oscillatory integrals and multipliers on $F L^{p}$, Ark. Mat. 11 (1973), 1-11. MR0340924(49:5674)

7. Sanghyuk Lee, Bilinear restriction estimates for surfaces with curvatures of different signs, Trans. Amer. Math. Soc. 358 (2006), 3511-3533. MR.2218987 (2007a:42023)

8. Sanghyuk Lee, Linear and bilinear estimates for oscillatory integral operators related to hypersurfaces, J. Funct. Anal. 241 (2006), 56-98. MR2264247 (2007g:42024)

9. Sanghyuk Lee and A. Vargas, Sharp null form estimates for the wave equation, Amer. J. Math. 130 (2008), 1279-1326. MR2450209 (2009k:35168)

10. G. Mockenhaupt, A note on the cone multiplier, Proc. Amer. Math. Soc. 117 (1993), 145-152. MR 1098404 (93c:42015)

11. G. Mockenhaupt, A. Seeger, and C. Sogge, Local smoothing of Fourier integral operators and Carleson-Sjölin estimates, J. Amer. Math. Soc. 6 (1993), 60-135. MR.1168960 (93h:58150)

12. G. Mockenhaupt, Bounds in Lebesgue spaces of oscillatory integral operators, Habilitationsschrift, Universität Siegen, 1996.

13. C.D. Sogge, Propagation of singularities and maximal functions in the plane, Invent. Math. 104 (1991), 329-376. MR1098614 (92i:58192)

14. C.D. Sogge, Fourier Integrals in Fourier analysis, Cambridge Tracts in Math., vol. 105, Cambridge Univ. Press, Cambridge, 1993. MR1205579 (94c:35178)

15. E.M. Stein, Harmonic Analysis: Real-Variable Methods, Orthogonality and Oscillatory Integrals, Princeton Univ. Press, Princeton, NJ, 1993. MR.1232192 (95c:42002) 
16. R.S. Strichartz, Restriction of Fourier transform to quadratic surfaces and decay of solutions of wave equations, Duke Math. J. 44 (1977), 705-774. MR0512086 (58:23577)

17. T. Tao, A. Vargas, and L. Vega, A bilinear approach to the restriction and Kakeya conjectures, J. Amer. Math. Soc. 11 (1998), 967-1000. MR.1625056 (99f:42026)

18. T. Tao and A. Vargas, A bilinear approach to cone multipliers I. Restriction estimates, Geom. Funct. Anal. 10 (2000), 185-215. MR.1748920(2002e:42012)

19. T. Tao and A. Vargas, A bilinear approach to cone multipliers II. Application, Geom. Funct. Anal. 10 (2000), 216-258. MR.1748921 (2002e:42013)

20. T. Tao, Endpoint bilinear restriction theorems for the cone, and some sharp null form estimates, Math. Zeitschrift 238 (2001), 215-268. MR1865417(2003a:42010)

21. T. Tao, A sharp bilinear restriction estimate for paraboloids, Geom. Funct. Anal. 13 (2003), 1359-1384. MR2033842 (2004m:47111)

22. A. Vargas, Restriction theorems for a surface with negative curvature, Math. Zeitschrift 249 (2005), 97-111. MR2106972 (2005f:42029)

23. T. Wolff, A sharp bilinear cone restriction estimate, Annals of Math. 153 (2001), 661-698. MR 1836285 (2002j:42019)

Department of Mathematics, Pohang University of Science and Technology, HyojaDong Nam-Gu, Pohang 790-784, Gyungbuk, Korea

Current address: Korea Institute for Advanced Study, 207-43 Cheongryangri 2-dong Dongdaemun-gu, Seoul 130-722, Korea

E-mail address: leejj@kias.re.kr 